\title{
Random Iterations of Subhyperbolic Relaxed Newton's Methods
}

\author{
Dissertation \\ zur Erlangung des Doktorgrades der \\ Mathematisch-Naturwissenschaftlichen Fakultäten der \\ Georg-August-Universität zu Göttingen
}

\author{
Vorgelegt von \\ Ghazaleh Arghanoun
}

aus

Teheran, Iran

Göttingen 2004 
D7

Referent: PD Dr. Hartje Kriete

Korreferent: Prof. Dr. Manfred Denker

Tag der mündlichen Prüfung: 14.04.2004 


\section{Acknowledgement}

I would like to acknowledge all the people who have contributed to the improvement of this thesis through their critisisms, suggestions and patience; special thanks are due to my Advisor PD Dr. Hartje Kriete for his extensive support. He helped me with several corrections and motivating discussions. I would also like to thank Prof. Dr. Siavash Shahshahaani. A large part of my knowledge in the general theory of dynamical systems is due to him. Also, I want to express my sincerest thanks to my parents and Arne Ackermann. 


\section{Contents}

\begin{tabular}{lll}
\hline 1 & Preface & IV
\end{tabular}

$\begin{array}{lll}2 & \text { Known Results } & 1\end{array}$

2.1 Preliminaries . . . . . . . . . . . . . . . . . . . 1

2.1.1 The Extended Complex Plane . . . . . . . . . . . . . 1

2.1 .2 Rational Functions . . . . . . . . . . . . . . . . 4

$2.2 \quad$ Iteration of One Rational Map . . . . . . . . . . . . . . . 11

2.3 Random Iterations: General Case . . . . . . . . . . . . . 27

3 The Subhyperbolic Relaxed Newton's Method 33

3.1 Introductory Remarks . . . . . . . . . . . . . . . . . 33

3.2 Near $\left(\mathbf{P}_{\mathbf{0}}, \mathbf{h}_{\mathbf{0}}\right) \ldots \ldots \ldots \ldots \ldots$

$3.2 .1 \quad$ Introduction . . . . . . . . . . . . . . . 36

3.2 .2 The Julia Set . . . . . . . . . . . . . . . . . . 36

3.2 .3 The Stable Domains . . . . . . . . . . . . . . . . . . 42

3.3 Convergent Sequences $\operatorname{Near}\left(\mathbf{P}_{\mathbf{0}}, \mathbf{h}_{\mathbf{0}}\right)$. . . . . . . . . . . 48

$3.3 .1 \quad$ Introduction . . . . . . . . . . . . . . . . . . . . 48

3.3 .2 The Structure of the Fatou Set . . . . . . . . . . . . . 49

3.3 .3 The Measure of the Julia Set . . . . . . . . . . . . . . 56

3.4 A General Convergent Sequence . . . . . . . . . . . . . . 61

3.4 .1 Introduction . . . . . . . . . . . . . . . . . 61

3.4 .2 The Fatou Set . . . . . . . . . . . . . . . 61

3.4 .3 The Julia Set . . . . . . . . . . . . . . . . . . . 64

3.4 .4 Connectedness of the Julia Set . . . . . . . . . . . 65

4 An Application: the Newton's Petals 69

4.1 Basic Assumptions . . . . . . . . . . . . . . . . . . . . . . . . 69 
4.2 Virtual Petals . . . . . . . . . . . . . . . . . . . . . . . . . 71

4.2 .1 Quasiconformal Mappings . . . . . . . . . . . . 71

4.2 .2 The Process of Approximating . . . . . . . . . . . . . 74

$\begin{array}{llr}5 & \text { References } & 80\end{array}$ 


\section{Preface}

The theory of polynomial functions is one of the oldest constituents of modern mathematics. Numerous attempts in the course of history have been leading to versatile methods for studying the different aspects of such functions, among them methods for extending their domains of definition, which play an important role in various branches of mathematics like the theory of the Riemannian surfaces and differential topology, as well as several iterating processes for locating their zeros, which once perhaps motivated by approximating an irrational number by rational ones, come partly from the theory of ordinary differential equations and dynamical systems.

Among the different kinds of iterating methods, the one which is of interest to us is the so-called relaxed Newton's method. Its basic idea comes from a process known as Euler's method for approximating the solutions of the initial value problems.

If we look at the differential equation

$$
z^{\prime}(t)=f(z(t))=f(t, z)
$$

where $t$ is a real number, and $z$ takes values from the set of complex numbers, then solving this differentail equation is geometrically equivalent to determining a curve $z=z(t)$ which passes through a given initial point $z\left(t_{0}\right)=z_{0}$ and has its slope at each point equal to the value prescribed by the function $f$ applied to that point. Suppose now that we are at the initial point $z_{0}$. As $t$ increases by a small step $h$, we move along the tangent line in 
the direction of $f\left(z_{0}\right)=f\left(t_{0}, z_{0}\right)$ to the point $z_{1}=z_{0}+h f\left(t_{0}, z_{0}\right)$, which is the result of truncating of the actual solution

$$
z\left(t_{1}\right)=z_{0}+h f\left(t_{0}, z_{0}\right)+\frac{1}{2} h^{2} z^{\prime \prime}\left(t_{0}\right)+\cdots, \quad t_{1}=t_{0}+h
$$

after the linear term in $h$.

If the step size $h>0$ is small enough, and if the slope is not changing too drastically near $\left(t_{0}, z_{0}\right)$, the value $z_{1}$ will be close to $z\left(t_{1}\right)$. In the same way we can start from $z_{1}$, using the slope given by $f\left(t_{1}, z_{1}\right)$, to get

$$
\left(t_{2}, z_{2}\right)=\left(t_{1}+h, z_{1}+h f\left(t_{1}, z_{1}\right)\right)
$$

Actually, we are going through an iterating process defined by

$$
\left\{\begin{aligned}
t_{n} & =t_{n-1}+h=t_{0}+n h \\
z_{n} & =z_{n-1}+h f\left(t_{n-1}, z_{n-1}\right)
\end{aligned}\right.
$$

to approximate the solution $z(t)$ of our differential equation by $z_{n}$ 's.

The first formal description of this method is attributed to Euler, but the first proof of the fact that the approximate solution $z_{n}$ converges to a solution as the step $h$ gets smaller is due to Cauchy; Newton had also used this method without comment in the very first book using differential equations. Indeed, one can use an algorithm based on this method to approximate the solutions to the differential equation $x^{\prime}=r x$ for real valued $x$, which match some tables of calculations dating back to babylonians of 2000 B.C.. Henrici, Hubbard-West

Let us assume a fixed polynomial $P_{0}$ defined on the Riemann sphere $\overline{\mathbf{C}}$, $P_{0}: \overline{\mathbf{C}} \longrightarrow \overline{\mathbf{C}}$. If we apply first the formula (1) to the rational function $f(z)=-\frac{P_{0}(z)}{P_{0}^{\prime}(z)}$, we get the so-called Newton flow 


$$
\text { (3) } \quad z^{\prime}(t)=-\frac{P_{0}(z)}{P_{0}^{\prime}(z)}
$$

for the complex polynomial $P_{0}(z)$. A zero of $P_{0}$ is called an equilibrium of (3), and a zero of $P_{0}^{\prime}$ which is not also a zero of $P_{0}$ is a critical point of (3) Benzinger. Substution $w=P_{0}(z)$ leads to the flow

$$
w^{\prime}=-w
$$

which is one of the simplest linear systems in $\mathbf{R}^{2} \cup\{\infty\}$. After solving,

$$
w(t)=\left(w_{1}(t), w_{2}(t)\right)=\left(c_{1} e^{-t}, c_{2} e^{-t}\right),
$$

and we recognize a stable node (sink) at the origin and an unstable node (source) at $\infty$ (see for example [Perko, chap. 1]). Indeed,

$$
P_{0}(z(t))=P_{0}\left(z_{0}\right) e^{-t}
$$

which means the solutions of the main system are the inverse images of the lines $\theta=\theta_{0}, \theta_{0} \in[0,2 \pi)$, under $P_{0}$. Further, the zeros of $P_{0}(z)$ are all sinks, and $\infty$ is a source of the Newton flow, respectively. Haeseler-Kriete]

What we mean by a relaxed Newton's method is now the discretized Euler's method related to the Newton flow (3). In our case, we choose a small step $0<h_{0} \leq 1$ and apply the second formula of $(2)$ to the function $f(z)=\frac{P_{0}(z)}{P_{0}^{\prime}(z)}$ to obtain

$$
N_{P_{0}, h_{0}}(z)=z-h_{0} \frac{P_{0}(z)}{P_{0}^{\prime}(z)}
$$

The function $N_{P_{0}, h_{0}}: \overline{\mathbf{C}} \longrightarrow \overline{\mathbf{C}}$ is called the relaxed Newton's method for the polynomial $P_{0}$. For an initial value $z_{0}$ the iterating process is then defined by

$$
z_{n+1}=N_{P_{0}, h_{0}}\left(z_{n}\right), \quad n \geq 0 .
$$


The set $\left\{z_{n}\right\}_{n \geq 0}$ is called the (forward) orbit of the initial point $z_{0}$ under the family $\left\{N_{P_{0}, h_{0}}^{n}\right\}_{n \geq 0}$, where $N_{P_{0}, h_{0}}^{n}=\overbrace{N_{P_{0}, h_{0}} \circ \cdots \circ N_{P_{0}, h_{0}}}^{n-\text { times }}$. This discrete system divides the Riemann sphere into two sets: the open Fatou set $\mathbf{F}\left(N_{P_{0}, h_{0}}\right)$, where the family $\left\{N_{P_{0}, h_{0}}^{n}\right\}_{n \geq 0}$ is normal, and the orbits of the points of each connected component of $\mathbf{F}\left(N_{P_{0}, h_{0}}\right)$ move stabely according to some prescribed pattern, and the closed perfect Julia set, where the orbits of the points behave chaotic. The study of the orbits of the points $z_{0} \in \overline{\mathbf{C}}$ under $\left\{N_{P_{0}, h_{0}}^{n}\right\}_{n \geq 0}$ falls within the scope of the classical theory of iterations of one rational map [Beardon, Milnor, Carleson-Gamelin]. As one may expect, there are qualitative similarities between these orbits and the trajectories of the Newton flow as $h \longrightarrow 0$ ([ Kriete, Haeseler-Kriete, Benzinger $)$ :

Theorem 1 Haeseler-Kriete, Theorem B] Let $P$ be a polynomial, $\operatorname{deg}(P) \geq$ 2 , and $\Gamma$ be the union of all trajectories which reach some critical point of the Newton flow (3) in finite time. Then for $h \longrightarrow 0$ :

- the Julia sets of $N_{P, h}$ tend to $\Gamma$ (with respect to the Hausdorff metric)

- for every $z_{0} \notin \Gamma$ the orbits $\left\{N_{P, h}^{n}\left(z_{0}\right)\right\}_{n \geq 0}$ of $z_{0}$ under the families $\left\{N_{P, h}^{n}\right\}_{n \geq 0}$ tend to its trajectory $\left\{z_{z_{0}}(t) \mid t \geq 0\right\}$ under the Newton flow (with respect to the Hausdorff metric).

While trying to trace the trajectories of the Newton flow or even the discrete dynamical system produced by iterating the relaxed Newton's method of a polynomial as above on the monitor of a computer one confronts some distortions in the dynamics of the orbits which emerge from two main error sources: the truncation of the Taylor series for a solution, and the limitations of finite accuracy due to the computing on actual machines. In order to providing better aproximations to the orbits, one can consider other families of 
rational maps, e. g. $\left\{N_{P_{n}, h_{n}} \circ \cdots \circ N_{P_{1}, h_{1}}\right\}_{n \in \mathbf{N}}$, where $\left\{P_{n}\right\}_{n \in \mathbf{N}}$ is a set of polynomials "suitabely close" to an initial polynomial $P_{0},\left\{h_{n}\right\}$ is a sequence of real numbers in $(0,1]$, and $N_{P_{i}, h_{i}}$ is the relaxed Newton's method defined for $\left(P_{i}, h_{i}\right)$.

This approach will be developed in this paper for an initial polynomial $P_{0}$ and sequences of polynomials $\left\{P_{n}\right\}$, which satisfy certain conditions, and sequences $\left\{h_{n}\right\}$ of positive real numbers in a small neighborhood of some fixed number $0<h_{0} \leq 1$.

The next chapter gives an overview of the main results already known in the theory of the standard and random iterations of rational maps.

In chapter 3 we begin our study of random iterations of the relaxed Newton's methods $\left\{N_{n}\right\}$ explained above near a given pair $\left(P_{0}, h_{0}\right)$ with the property that the rational function $N_{P_{0}, h_{0}}$ produced by $\left(P_{0}, h_{0}\right)$ is a subhyperbolic rational function. We restrict ourselves first to the small neighborhoods of $\left(P_{0}, h_{0}\right)$ and show that the Julia set of the family $\left\{N_{n}\right\}$ is still a nonempty, perfect, nowhere dense set (Theorem 1 and 2, Lemma 1, sec. 3.2.2), and there is no "wandering domains" among the connected components of its Fatou set (the last paragraph of sec. 3.2.3). Then we consider arbitrary sequences $\left\{\left(P_{i}, h_{i}\right)\right\}_{i \in \mathbf{N}}$ convergent to $\left(P_{0}, h_{0}\right)$ in such neighborhoods and prove that the Julia set of $\left\{N_{n}\right\}$ is a connected and locally connected closed perfect nowhere dense set of measure zero (sec. 3.3.3 and Theorems 9 and 10, sec. 3.4.4), and the Fatou set consists of contracting domains (Theorem 6, sec. 3.2.2). At last, we deduce the same results for a general sequence $\left\{\left(P_{i}, h_{i}\right)\right\}_{i \in \mathbf{N}}$ convergent to $\left(P_{0}, h_{0}\right)$ (sec. 3.3).

Chapter 4 gives an application of subhyperbolic families of relaxed Newton's methods to the case when $N_{P_{0}, h_{0}}$ has a parabolic periodic point, and therefore not any more a subhyperbolic rational function. Using a special method from the theory of quasiconformal mappings known as "quasiconfor- 
mal surgery" we show how the parabolic periodic point and its basin can be approximated by means of a family of random iterations of definite subhyperbolic relaxed Newton's methods which converge to $N_{P_{0}, h_{0}}$. Hence we turn this case to the one already studied in chapter 3 . 


\section{Known Results}

\subsection{Preliminaries}

\subsubsection{The Extended Complex Plane}

The set of complex numbers $\mathbf{C}=\{z=x+i y \mid x$ and $y \in \mathbf{R}\}$ has many advantages when used as the domain of definition of a function. One can see for example that $\mathbf{C}$ is algebarically closed, i. e. every polynomial with complex coefficients has at least one root in $\mathbf{C}$. On the other hand, one can give $\mathbf{C}$ some topological structure by considering it as the Euclidean plane $\mathbf{R}^{2}$. The set $\mathbf{C}$ regarded in this way is called the complex plane. This is a metric space with the usual distance between two points $z_{1}=x_{1}+i y_{1}$ and $z_{2}=x_{2}+i y_{2}$ defined by

$$
d\left(z_{1}, z_{2}\right)=\left(\left(x_{1}-x_{2}\right)^{2}+\left(y_{1}-y_{2}\right)^{2}\right)^{\frac{1}{2}}
$$

There are also disadvantages of using $\mathbf{C}$ as the domain of definition, among them that $\mathbf{C}$ is not compact, or equivalently not every sequence of points in $\mathbf{C}$ has a convergent subsequence. On the other hand, division by 0 is impossible, and some functions are not decined every where, for example $f(z)=z^{-1}$ is not defined at 0 . To avoid these disadvantages, we introduce the set $\overline{\mathbf{C}}=\mathbf{C} \cup\{\infty\}$, where $\infty$ (called the point at infinity) is a symbol which does not represent an element of $\mathbf{C}$. We call the set $\overline{\mathbf{C}}$ the extended complex plane.

To define a suitable topology on $\overline{\mathbf{C}}$, we notice first that there is a welldefined map between the 2-sphere $\mathbf{S}^{\mathbf{2}}=\left\{\left(x_{1}, x_{2}, x_{3}\right) \in \mathbf{R}^{\mathbf{3}} \mid x_{1}^{2}+x_{2}^{2}+x_{3}^{2}=1\right\}$ 
and the extended complex plane $\overline{\mathbf{C}}$, known as the stereographic projection $\pi: \mathbf{S}^{2} \longrightarrow \overline{\mathbf{C}}$ defined by

$$
\left\{\begin{array}{l}
\pi\left(x_{1}, x_{2}, x_{3}\right)=\left(x\left(x_{1}, x_{2}, x_{3}\right), y\left(x_{1}, x_{2}, x_{3}\right)\right), \text { if }\left(x_{1}, x_{2}, x_{3}\right) \neq(0,0,1) \\
\pi(0,0,1)=\infty
\end{array}\right.
$$

where

$$
\begin{aligned}
& x\left(x_{1}, x_{2}, x_{3}\right)=\frac{x_{1}}{1-x_{3}} \\
& y\left(x_{1}, x_{2}, x_{3}\right)=\frac{x_{2}}{1-x_{3}} .
\end{aligned}
$$

This map is one to one and onto and transfers the Euclidean metric of $\mathbf{S}^{2}$ (induced from $\mathbf{R}^{3}$ ) to a metric $\sigma$ on $\overline{\mathbf{C}}$ defined by

$$
\sigma(z, w)=\left|\pi^{-1}(z)-\pi^{-1}(w)\right|
$$

We get therefore an explicit formula for $\sigma$ :

$$
\left\{\begin{array}{l}
\sigma(z, w)=\frac{|z-w|}{\left(1+|z|^{2}\right)^{\frac{1}{2}}\left(1+|w|^{2}\right)^{\frac{1}{2}}}, \quad \text { if } z \text { and } w \in \mathbf{C}, \\
\sigma(z, \infty)=\lim _{w \rightarrow \infty} \sigma(z, w)=\frac{2}{\left(1+|z|^{2}\right)^{\frac{1}{2}}} .
\end{array}\right.
$$

The metric $\sigma$ is called the chordal metric on $\overline{\mathbf{C}}$ since $\sigma(z, w)$ is the Euclidean length of the chord joining $\pi^{-1}(z)$ and $\pi^{-1}(w)$ in $\mathbf{S}^{2}$. It converts $\overline{\mathbf{C}}$ to a metric space so that the stereographic projection $\pi$ defines a homeomorphism between the 2-sphere $\mathbf{S}^{2}$ and the extended complex plane $\overline{\mathbf{C}}$, i. e. the map $\pi$ and its inverse $\pi^{-1}: \overline{\mathbf{C}} \longrightarrow \mathbf{S}^{2}$ are both continuous Jones-Singerman, chap. 1]. 
The space $\overline{\mathbf{C}}$ with the topology induced by the metric just defined on it is indeed nothing else than the one-point compactification of $\mathbf{C}$ [Munkres]. Hence $\overline{\mathbf{C}}$ is compact, and every open set $U$ in $\overline{\mathbf{C}}$ is either open in $\mathbf{C}$ or the complement of a compact subset $K \subset \mathbf{C}$ in $\overline{\mathbf{C}}, U=\overline{\mathbf{C}} \backslash K$. We are now able to recognize among others special subsets of $\overline{\mathbf{C}}$ known as domains which are nonempty, path-connected, open subsets of $\overline{\mathbf{C}}$.

There is another metric on $\overline{\mathbf{C}}$, equivalent to the chordal metric, known as the spherical metric $\chi$. The spherical distance $\chi(z, w)$ between $z$ and $w$ in the extended plane is by definition the Euclidean length of the shortest path on $\mathbf{S}^{2}$ between $\pi^{-1}(z)$ and $\pi^{-1}(w)$. The both metrics relate to each other by the formula

$$
\sigma(z, w)=2 \sin \left(\frac{\chi(z, w))}{2}\right)
$$

which yields the inequalities

$$
\left(\frac{2}{\pi}\right) \chi(z, w) \leq \sigma(z, w) \leq \chi(z, w)
$$

i. e. the two metrics $\chi$ and $\sigma$ are equivalent, and the topological structures produced by them on $\overline{\mathbf{C}}$ are the same. [Beardon, sec. 2.1]

If we pull the spherical metric $\chi$ "locally" from an open subset $U \subset$ $\overline{\mathbf{C}}$ back to its preimage $V \subset \mathbf{R}^{\mathbf{2}}$ under an embedding $\eta$ which is either a restriction of stereographic projection $\pi$ to $U$, or the embedding $f \circ \pi$, where $f(z)=\frac{1}{z}$ in case $\infty \in U$, we obtain a conformal metric of the form $\gamma(z)|d z|=d s=2 \frac{|d z|}{\left(1+|z|^{2}\right)}$ on $V$. This enables us to define a bounded measure $\mu$ (by defining such concepts as length, area and so on) for the subsets of $\overline{\mathbf{C}}$ in the sense of Lebesgue measure on $\mathbf{R}^{\mathbf{2}}$. For example if we consider the above mentioned embedding for $U$ :

$$
\eta: V \longrightarrow U \subset \overline{\mathbf{C}}
$$


which pulls the spherical metric $\chi$ back to $V$ in the form of the metric $\gamma(z)|d z|=2 \frac{|d z|}{\left(1+|z|^{2}\right)}$, the "area" of $V$ in this metric is by definition the integral

$$
\mathcal{A}=\iint_{V} \gamma(x+i y)^{2}|d x||d y|
$$

[Milnor, p. 21, chap. 17]

\subsubsection{Rational Functions}

Let $\mathcal{M}(\overline{\mathbf{C}}, \overline{\mathbf{C}})$ be the class of all functions from $\overline{\mathbf{C}}$ to itself ( we recall that a map $f$ is said to be defined near $\infty$ if it is defined on some subset $\{|z|>$ $r\} \cup\{\infty\}$ which is as mentioned above an open neighborhood of $\infty)$. We define a metric on $\mathcal{M}(\overline{\mathbf{C}}, \overline{\mathbf{C}})$ by

$$
\begin{aligned}
& \rho(f, g)=\sup \{\sigma(f(z), g(z)) \mid z \in \overline{\mathbf{C}}\} \\
& \forall f, g \in \mathcal{M}(\overline{\mathbf{C}}, \overline{\mathbf{C}}) .
\end{aligned}
$$

The metric $\rho$ is called the uniform metric (or the metric of uniform convergence) on $\mathcal{M}(\overline{\mathbf{C}}, \overline{\mathbf{C}})$ ([Ahlfors, Munkres, Beardon] $)$. We could equally well use the spherical instead of the chordal metric to define the uniform metric on $\mathcal{M}(\overline{\mathbf{C}}, \overline{\mathbf{C}})$.

The convergence in the metric space $\mathcal{M}(\overline{\mathbf{C}}, \overline{\mathbf{C}})$ equipted with $\rho$ can be defined as usual:

Definition 1 A sequence of elements $\left\{f_{n}\right\}_{n \in \mathbf{N}} \subset \mathcal{M}(\overline{\mathbf{C}}, \overline{\mathbf{C}})$ converges to some $f \in \mathcal{M}(\overline{\mathbf{C}}, \overline{\mathbf{C}})$ with respect to the uniform metric, if for every $\epsilon>0$ there exists some positive integer $n_{0}$ so that

$$
\rho\left(f_{n}, f\right)<\epsilon \text {, whenever } n>n_{0} \text {. }
$$


We note that a map $f \in \mathcal{M}(\overline{\mathbf{C}}, \overline{\mathbf{C}})$ is continuous at $\infty$ if the map $z \longmapsto$ $f\left(\frac{1}{z}\right)$ is continuous at 0 . The subclass $\mathcal{C}(\overline{\mathbf{C}}, \overline{\mathbf{C}})$, in short $\mathcal{C}$, is by definition the class of all continuous maps of $\overline{\mathbf{C}}$ to itself. This subclass is actually a closed subset of $\mathcal{M}(\overline{\mathbf{C}}, \overline{\mathbf{C}})$, which means the limit of every convergent sequence of continuous functions on $\overline{\mathbf{C}}$ is itself a continuous function on $\overline{\mathbf{C}}$. Munkres, Ahlfors

Now we concentrate on $\mathcal{C}$.

Definition 2 A family $\mathcal{F} \subset \mathcal{M}(\overline{\mathbf{C}}, \overline{\mathbf{C}})$ is equicontinuous at $z_{0} \in \overline{\mathbf{C}}$ if and only if for every positive $\epsilon$ there is a positive $\delta$ such that for all $z \in \overline{\mathbf{C}}$ and for all $f \in \mathcal{F}$,

$$
\sigma\left(z_{0}, z\right)<\delta \Longrightarrow \sigma\left(f\left(z_{0}\right), f(z)\right)<\epsilon
$$

The family $\mathcal{F}$ is equicontinuous on a subset $U$ of $\overline{\mathbf{C}}$ if it is equicontinuous at every point of $U$. Beardon]

From the above definition we see that every equicontinuous family is actually a subset of $\mathcal{C}$. A map $f$ in $\mathcal{C}$ is holomorphic in a domain $D \subset \overline{\mathbf{C}}$ if the derivative $f^{\prime}$ exists and is bounded at each point of $D$. The map $f$ is meromorphic in $D$ if each point of $D$ has a neighborhood on which either $f$ or $\frac{1}{f}$ is holomorphic. A pole of $f$ is a point $w$ where $f(w)=\infty$. Near such a point $w$ the map $z \longmapsto \frac{1}{f(z)}$ is holomorphic with value 0 at $w$. Indeed, $\frac{1}{f}$ is continuous at $w$ in the Euclidean metric, hence in chordal metric. Since $\sigma\left(\frac{1}{z}\right)=\sigma(z)$

$$
\sigma(f(z), f(w))=\sigma\left(\frac{1}{f(z)}, \frac{1}{f(w)}\right)=\sigma\left(\frac{1}{f(z)}, 0\right) \longrightarrow 0, \text { as } z \longrightarrow w
$$

A complex polynomial $P: \overline{\mathbf{C}} \longrightarrow \overline{\mathbf{C}}$, is a function of the form 


$$
P(z)=a_{0}+a_{1} z+a_{2} z^{2}+\cdots+a_{n} z^{n}
$$

where $n$ is a positive integer, and $a_{0}, \cdots, a_{n}$ are complex numbers. The number $n \geq 0$ is called the degree of the polynomial $P$ : $\operatorname{deg}(P)=n$.

A rational function $R: \overline{\mathbf{C}} \longrightarrow \overline{\mathbf{C}}$ is a function of the form $R(z)=\frac{P(z)}{Q(z)}$, where $P$ and $Q$ are both polynomials as defined above, where $P(z)$ and $Q(z)$ are not both being the zero polynomial. If $P \equiv 0(Q \equiv 0)$ then $R$ is the constant function $0(\infty)$.

If a function $R: \overline{\mathbf{C}} \longrightarrow \overline{\mathbf{C}}$ is rational then it is meromorphic on $\overline{\mathbf{C}}$; conversely, each meromorphic map on $\overline{\mathbf{C}}$ is a rational function. Jones-Singerman, Milnor

The rational funtion $R$ is not defined at the common zeros of $P$ and $Q$. If there is some common zero, we may cancel the corresponding linear factors and thereby assume that $P$ and $Q$ are coprime, i.e. they have no common zeros. We assume always that this has been done. The degree $\operatorname{deg}(R)$ of $R$ is then defined by

$$
\operatorname{deg}(R)=\max \{\operatorname{deg}(P), \operatorname{deg}(Q)\}
$$

where $\operatorname{deg}(S)$ is the degree of a given polynomial $S$. If $R$ is a constant map with the constant value $\alpha \in \overline{\mathbf{C}}$, then $\operatorname{deg}(R)=0$.

Theorem 1 Suppose that $R_{n}$ is meromorphic in a domain $D$ on $\overline{\mathbf{C}}$ and $R_{n}$ converges to $R$ with respect to uniform metric $\rho$ restricted to $\mathcal{C}(D, \overline{\mathbf{C}})$. Then $R$ is meromorphic in D, too. [Beardon, p. 46]

Therefore the subclass $\mathcal{R} \subset \mathcal{C}$ of all rational functions on $\overline{\mathbf{C}}$ is a closed subset of $\mathcal{C}$ with respect to the topology induced by the metric $\rho$.

Let us consider now the function 


$$
\operatorname{deg}: \mathcal{R} \longrightarrow \mathbf{Z}^{+}
$$

which maps each rational map $R$ to its degree $\operatorname{deg}(R)$. This function enables us to deduce some facts about the connected components of the metric space $\mathcal{R}$.

Theorem 2 The map deg $: \mathcal{R} \longrightarrow \mathbf{Z}^{+}$is continuous. In particular, if the rational functions $R_{n}$ converge in uniform metric to some function $R$, then $R$ is rational and for sufficiently large $n, \operatorname{deg}\left(R_{n}\right)=\operatorname{deg}(R)$. Beardon, $p$. $46]$

The class $\mathcal{R}_{n}$ of rational maps of degree $n$ is the inverse image of the open and closed set $\{n\} \subset \mathbf{Z}^{+}$under the continuous map deg. Hence $\mathcal{R}_{n}$ is an open and closed subset of $\mathcal{R}$. On the other hand we can always slide zeros and poles of $R \in \mathcal{R}_{n}$ to those of $S \in \mathcal{R}_{n}$ maintaining the same degree ([Ahlfors]). Thus each $\mathcal{R}_{n}$ is connected and $\left\{\mathcal{R}_{n}\right\}$ make the connected components of $\mathcal{R}$ with respect to uniform metric $\rho$.

Each $R \in \mathcal{R}_{n}$ of degree $d$ determines its $d$ zeros and $d$ poles (counted with multiplicity) uniquely, so it determines its coefficients up to scalar multiples, i.e. there is a map

$$
\psi: \mathcal{R}_{d} \longrightarrow \mathbf{C P}^{2 d+1}
$$

from $\mathcal{R}_{d}$ to the complex projective space obtained form $\mathbf{C}^{2 d+2}-\{0\}$ by identification of the vectors which are nonzero scalar multiples of each other. The map $\psi$ is actually a homeomorphism of $\mathcal{R}_{d}$ onto its image $\psi\left(\mathcal{R}_{d}\right)=$ $\left\{\left(x_{0}: \cdots: x_{d}: y_{0}: \cdots: y_{d}\right) \in \mathbf{C P}^{2 d+1} \mid x_{d} \neq 0\right.$ or $\left.y_{d} \neq 0\right\}$ with the induced topology from $\mathbf{C P}^{2 d+1}$. The image $\psi\left(\mathcal{R}_{d}\right)$ is an open dense subset of $\mathbf{C P}^{2 d+1}$. [Beardon, p. 47] 
As we mentioned, if $\left\{R_{n}\right\} \subset \mathcal{R}_{d}$ is a sequence of meromorphic functions on $\overline{\mathbf{C}}$ which converges to some function $R$ in uniform metric, then $R$ is also meromorphic on $\overline{\mathbf{C}}$. The convergence of $\left\{R_{n}\right\}$ in $\mathcal{R}_{d}$ can be interpreated as a kind of convergence of the sequence of functions $\left\{R_{n}\right\}$ on $\overline{\mathbf{C}}$ which is known as the uniform convergence:

Definition 3 The sequence of the maps $\left\{f_{n}\right\}_{n \in \mathbf{N}}$ is uniformly convergent to a map $f$ on a domain $D \subseteq \overline{\mathbf{C}}$ if for each $\varepsilon>0$ there is a positive integer $N$ such that for every $n \geq N$ and each $x \in D, \sigma\left(f_{n}(x)-f(x)\right)<\varepsilon$.

This definition of convergence is evidently stronger than the pointwise convergence of a family $\left\{f_{n}\right\}_{n \in \mathbf{N}}$ of functions to a limit function $f$ on a domain $D \subseteq \overline{\mathbf{C}}$. We recall that such a family converges pointwise to $f$ on $D$ if the sequence of complex numbers $\left\{f_{n}(z)\right\}_{n \in \mathbf{N}}$ converges to the complex number $f(z)$, for every point $z \in D$.

For our purposes we need also a weaker form of the uniform convergence:

Definition 4 A sequence $\left\{f_{n}\right\}_{n \in \mathbf{N}}$ of maps on $\overline{\mathbf{C}}$ converges localy uniformly on some domain $D$ of $\overline{\mathbf{C}}$ to some map $f$ if each point $x$ in $D$ has a neighborhood on which $\left\{f_{n}\right\}$ converges uniformly to $f$. In this case, the convergence is uniform on each compact subset of $D$.

Definition 5 A family $\mathcal{F} \subset \mathcal{C}$ is called a normal family on a domain $D \subset \overline{\mathbf{C}}$ if every sequence of maps $\left\{f_{n}\right\}_{n \in \mathbf{N}} \subset \mathcal{F}$ has a subsequence which converges locally uniformly to some limit map $f$ on $D$.

Theorem 3 (Arzelà-Ascoli Theorem) Let $D \in \overline{\mathbf{C}}$ be a domain, and $\mathcal{F}$ be a family of continuous maps in $\mathcal{C}$. Then $\mathcal{F}$ is equicontinous in $D$ if and only if it is a normal family on D. Ahlfors, p. 222] 
Let us further assume $\mathcal{F} \subset \mathcal{R}$ to be a set of the form $\left\{f_{n}\right\}_{n \in \mathbf{N}}$ of rational functions $f_{n}$.

\section{Definition 6}

- The set

$\mathbf{F}(\mathcal{F}):=\left\{z \in \overline{\mathbf{C}} \mid\left\{\left.f_{n}\right|_{U}\right\}_{n \in \mathbf{N}}\right.$ is normal in a neighborhood $U$ of $\left.z\right\}$

is called the Fatou set of the family $\mathcal{F}$.

- The set $\mathbf{J}(\mathcal{F}):=\overline{\mathbf{C}} \backslash \mathbf{F}(\mathcal{F})$ is called the Julia set of the family $\mathcal{F}$.

From the definition, the set $\mathbf{F}(\mathcal{F})$ is an open set and hence $\mathbf{J}(\mathcal{F})$ is a closed one. Therefore the connected components of $\mathbf{F}(\mathcal{F})$ are domains:

Definition 7 The connected components of $\mathbf{F}(\mathcal{F})$ are called stable domains of $\mathcal{F}$.

Definition $8 \mathcal{G}(D)$ is by definition the set of limit functions of all possible convergent subsequences of $\mathcal{F}$ on a domain $D$. If all elements of $\mathcal{G}(D)$ are constant functions, the domain $D$ is called a contracting domain.

With this background, we are now able to state some standard theorems from complex analysis which play an important role in the next sections.

Theorem 4 (Theorem of Vitali) Suppose that the family $\mathcal{F}$ is normal in the domain $D$, and $f_{n}$ 's converge pointwise to a map $f$ on some nonempty open subset $W$ of $D$. Then there is a map $F$ meromorphic on $D$ such that $\left.F\right|_{W}=f$, and $f_{n} \longrightarrow F$ locally uniformly on D. [Beardon, $p$. 56] 
Theorem 5 (Theorem of Montel) Let $D$ be a domain on $\overline{\mathbf{C}}$, and let $\Omega$ be the domain $\overline{\mathbf{C}} \backslash\{0,1, \infty\}$. Then the family consisting of all analytic maps $f: D \longrightarrow \Omega$ is normal in D. [Beardon, $p$. 57]

There is a weeker version of Montel's theorem which we shall also need:

Theorem 6 (The weaker Theorem of Montel) Let $\mathcal{F}$ be a family of maps as before, each meromorphic in a domain D on the complex sphere. Suppose also there is a positive constant $m$ and, for each $f \in \mathcal{F}$, three distinct points $a_{f}, b_{f}$, and $c_{f}$ in $\overline{\mathbf{C}}$ such that:

1. $f \in \mathcal{F}$ does not take the values $a_{f}, b_{f}$, and $c_{f}$ in $D$; and

2. $\min \left\{\sigma\left(a_{f}, b_{f}\right), \sigma\left(b_{f}, c_{f}\right), \sigma\left(a_{f}, c_{f}\right)\right\} \geq m$.

Then $\mathcal{F}$ is normal in D. [Beardon, $p$. 57]

The next theorem known as Koebe's Distorsion Theorem enables us to deduce some facts about the measure (produced for example by the spherical metric on $\overline{\mathbf{C}}$ ) of the Julia set of definite families of rational functions:

Theorem 7 (Koebe's Distorsion Theorem) Let $D \subset \overline{\mathbf{C}}$ be a bounded region, and $E$ be a closed subset of $D$. There is a finite positive number $M$ for $E$ such that for every meromorphic function $R: D \longrightarrow \overline{\mathbf{C}}$ which is one to one on $D$, and each $z_{1}$ and $z_{2} \in E$ we have

$$
\frac{1}{M} \leq\left|\frac{R^{\prime}\left(z_{1}\right)}{R^{\prime}\left(z_{2}\right)}\right| \leq M
$$

[Golusin, p. 44] Carleson-Gamelin, sec. 1.1] Lyubich 1986] 


\subsection{Iteration of One Rational Map}

We consider first a family $\Upsilon=\left\{R^{n}\right\}_{n \in \mathbf{N}}$ of forward iterations $R^{n}$ of one

rational map $R$ of degree at least 2 on $\overline{\mathbf{C}}$. The set $\left\{R^{n}(z)\right\}_{n \in \mathbf{N}}$ is called the (forward) orbit of the point $z \in \overline{\mathbf{C}}$ under the family $\Upsilon$, as mentioned before. There are several standard results about the Fatou and Julia sets (cf. Def. 4) and the dynamical behavior of such families, some of which are listed below.

Theorem 8 Let $R$ be as above. The Fatou and Julia sets, $\mathbf{F}(\Upsilon)$ and $\mathbf{J}(\Upsilon)$ respectively, are completely invariant under $R$ :

$$
\begin{aligned}
& R(\mathbf{F}(\Upsilon))=\mathbf{F}(\Upsilon)=R^{-1}(\mathbf{F}(\Upsilon)) \\
& R(\mathbf{J}(\Upsilon))=\mathbf{J}(\Upsilon)=R^{-1}(\mathbf{J}(\Upsilon))
\end{aligned}
$$

[Beardon, p. 54]

Theorem 9 The Julia set $\mathbf{J}(\Upsilon)$ is nonempty. Furthermore it is a perfect set either equal to the whole $\overline{\mathbf{C}}$ or nowhere dense. [Beardon, p. 68]

Therefore the Julia set is uncountable.

Theorem 10 For $\Upsilon$ as above, let $W$ be any nonempty open set which meets $\mathbf{J}(\Upsilon)$. Then for all sufficiently large integers $n>0, \mathbf{J}(\Upsilon) \subset R^{n}(W)$. Beardon, p. 69]

Theorem 11 Let E be a closed, completely invariant subset of the complex sphere which has nonempty intersection with the Julia set $\mathbf{J}$. Then $E$ is infinite and $\mathbf{J} \subset E$. [Beardon, $p .67]$ 


\section{Definition 9}

- Let $R$ be as above. If there exists some $\alpha \in \overline{\mathbf{C}}$ and some positive integer $n>0$ such that $R^{n}(\alpha)=\alpha$ and $R^{m}(\alpha) \neq \alpha$ for $0<m<n$, then we call $\alpha$ a periodic point of (minimal) period $n$. The number $\left|\left(R^{n}\right)^{\prime}(\alpha)\right|$ is called the multiplier of $\alpha$. The set $\left\{\alpha, R(\alpha), \cdots, R^{n-1}(\alpha)\right\}$ is called a cycle of order $n$. A periodic point of order 1 is called a fixed point.

- If a point $\alpha \in \overline{\mathbf{C}}$ is not periodic, but for some natural number $m>0$, $R^{m}(\alpha)$ is periodic, then $\alpha$ is called a preperiodic point of $R$.

All of the periodic points of order $\mathrm{n}$ in a cycle $\left\{\alpha, R(\alpha), \cdots, R^{n-1}(\alpha)\right\}$ have the same multiplier $\left|\left(R^{n}\right)^{\prime}(\alpha)\right|$, according to the chain rule.

Definition 10 A periodic point $\alpha$ of order $n$ is called super-attracting, attracting, indifferent or repelling according as $\left|\left(R^{n}\right)^{\prime}(\alpha)\right|=0,<1,=1$ or $>1$, respectively.

Related to the last definition is the following

Theorem 12 The repelling periodic points of the family $\Upsilon$ are dense in $\mathbf{J}(\Upsilon)$. Beardon, p. 148]

Definition 11 Let the point $\alpha \in \overline{\mathbf{C}}$ be an indifferent periodic point of the family $\Upsilon$, and $\lambda$ be the multiplier of $\alpha$. If $\lambda$ is a root of unity, $\alpha$ is called a rationally indifferent periodic point. Otherwise $\alpha$ is an irrationally indifferent periodic point of $\Upsilon$.

Theorem 13 Every rationally indifferent periodic point of $\Upsilon$ lies in $\mathbf{J}(\Upsilon)$. [Beardon, p. 110]

From the definitions, every (super-)attracting periodic point of the family $\Upsilon$ is in the Fatou set $\mathbf{F}(\Upsilon)$. There are three different kinds of stable domains: 
Definition 12 Let $V$ be a stable domain of $\Upsilon$. Then

1. $V$ is periodic if for some $n \in \mathbf{N}, R^{n}(V) \cap V \neq \emptyset$;

2. $V$ is preperiodic if $V$ is not periodic but there exist $n, m \in \mathbf{N}$ with $n \neq m$ and $R^{n}(V) \cap R^{m}(V) \neq \emptyset$;

3. $V$ is wandering, if $V$ is neither periodic nor preperiodic.

Therefore, if a stable domain is not wandering, then it is either periodic or preperiodic:

Theorem 14 (Theorem of Sullivan) Consider the family $\Upsilon=\left\{R^{n}\right\}_{n \in \mathbf{N}}$ as before. There exists no wandering stable domain in the Fatou set of this family. Every periodic stable domain $V$ has one of the forms below:

1. Vhas a super-attracting periodic point $z_{0} \in V$ of order $p$, and $\lim _{n \rightarrow \infty} R^{n p}=$ $z_{0}$ locally uniformly on $V . V$ is called in this case a Boettcher domain.

2. Vhas an attracting periodic point $z_{0} \in V$ of order $p$, and $\lim _{n \rightarrow \infty} R^{n p}=$ $z_{0}$ locally uniformly on $V$. Furthermore, $R^{p}$ can be linearized in a neighborhood of $z_{0}$, i.e. there is a local diffeomorphism $g$ from a neighborhood of $z_{0}$ to a neighborhood of 0 such that $g \circ R^{p} \circ g^{-1}(z)=\lambda z$ with $0<\lambda<1$, for each $z$ in the above neighborhood of 0 . The real number $\lambda$ denotes the multiplier of $R^{p}$ at $z_{0}$. Such a $V$ is called a Schroeder domain.

3. The boundary $\partial V \subset \mathbf{J}(\Upsilon)$ of $V$ has a rationally indifferent periodic point $z_{0} \in \partial V$ of order $p$ such that $\lim _{n \rightarrow \infty} R^{n p}=z_{0}$, locally uniformly on $V$. Especially, $z_{0}$ belongs to $\mathbf{J}(\Upsilon)$. This $V$ is called a Leau domain associated with $z_{0}$. 
4. There is a conformal mapping $\phi: V \longrightarrow \boldsymbol{\Delta}$, where $\boldsymbol{\Delta}$ is the open unit disc $\{z \in \overline{\mathbf{C}}|| z \mid<1\}$, and an irrational number $\alpha \in \mathbf{R} \backslash \mathbf{Q}$ such that on $V$

$$
\phi\left(R^{p}\left(\phi^{-1}(z)\right)\right)=e^{2 \pi i \alpha} z .
$$

$V$ is called a Siegel disc. (Recall: a conformal mapping $\phi$ from a domain $V \subset \overline{\mathbf{C}}$ to a domain $W \subset \overline{\mathbf{C}}$ is an element of $\mathcal{C}(V, W)$ which is angleand direction-preserving.)

5. There is a conformal mapping $\varphi: V \longrightarrow \Lambda$, where $\Lambda:=\{z \in \mathbf{C} \mid 1<$ $|z|<r, r>0\}$, and an irrational number $\beta \in \mathbf{R} \backslash \mathbf{Q}$ such that on $V$

$$
\varphi\left(R^{p}\left(\varphi^{-1}(z)\right)\right)=e^{2 \pi i \beta} z .
$$

$V$ is called a Herman ring.

[Beardon, Milnor, Carleson-Gamelin]

\section{Definition 13}

- If $\alpha$ is a (super-)attracting fixed point of the family $\Upsilon$, the connected component of the Fatou set $\mathbf{F}(\Upsilon)$ which contains $\alpha$ is called the immediate basin of $\alpha$. If $B$ denotes this immediate basin, it follows that $R^{n}(z) \longrightarrow \alpha$ exatly when $z$ lies in some inverse image $R^{-n}(B)$, $m \geq 0$, of $B$. The set of such $z$ is called the basin of the attracting fixed point $\alpha$. More generally, the immediate basin of attraction of a (super-)attracting periodic point $\alpha$ of period $p$, which is also known as the immediate basin of attraction of the cycle $\left\{\alpha, R(\alpha), \cdots, R^{m-1}(\alpha)\right\}$, is the union of all (Boettcher) Schroeder domains which contain one of 
the elements of the cycle. The basin for such a periodic point (or its cycle) is the union of the immediate basin and all of its inverse images under $R$.

- If $\alpha$ is a rationally indifferent fixed point of the family $\Upsilon$, the union of those Leau domains associated with $\alpha$ is called the immediate basin of $\alpha$. The basin of $\alpha$ is the union of its immediate basin and all of the inverse images of this immediate basin under $R$. More generally, the immediate basin of a rationally indifferent periodic point $\alpha$ of period $m$, which is also known as the immediate basin of the cycle $\left\{\alpha, R(\alpha), \cdots, R^{m-1}(\alpha)\right\}$, is the union of all Leau domains associated with some element of the cycle. The basin for such a periodic point (or its cycle) is the union of its immediate basin and all of the preimages of this immediate basin under $R$.

If $\alpha$ is a (super-)attracting periodic point of period $m$ with the cycle $\left\{\zeta_{0}, \cdots, \zeta_{m-1}\right\}$, where $\zeta_{0}=\alpha$ and $\zeta_{i}=R^{i}(\alpha)$ for $i=1, \ldots, m-1$, then each $\zeta_{i}$ in the cycle belongs to a stable domain which is contained in the immediate basin of the cycle. For a rationally indifferent periodic point the situation is more complicated.

We first explain the dynamics near a rationally indifferent fixed point $\alpha$ which we suppose to be at 0 without loss of generality. For each positive $t$, each positive integer $p$ and each $k$ in $\{0,1, \cdots, p-1\}$ we define the sets

$$
\Pi_{k}(t)=\left\{r e^{i \theta}\left|r^{p}<t(1-\cos (p \theta)) ;\right| 2 k \pi / p-\theta \mid<\pi / p\right\}
$$

These sets are called petals at the origin ( Figure 1 for $p=4$ ). Beardon, p. 116] 


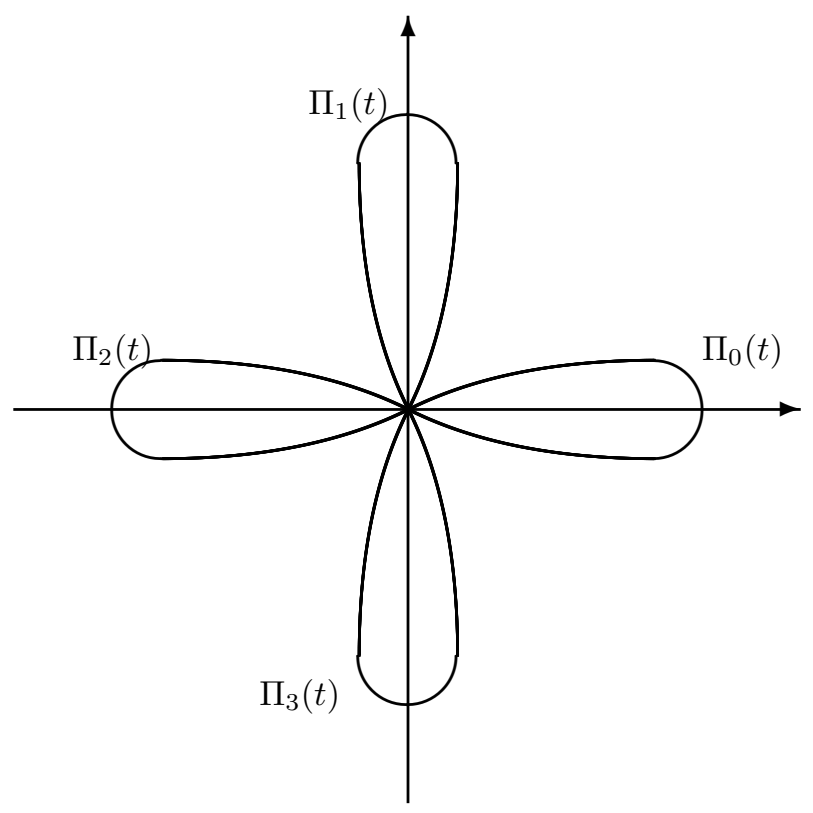

Figure 1

Four petals at the origin, for some $t>0$.

Theorem 15 (Petal Theorem) Suppose that the analytic map $R$ has a Taylor expansion

$$
R(z)=z-z^{p+1}+O\left(z^{2 p+1}\right)
$$

near 0 . Then for sufficiently small t:

1. $R$ maps each petal $\Pi_{k}(t)$ into itself (Figure 2);

2. $R^{n} \longrightarrow 0$ uniformly on each petal as $n \longrightarrow \infty$ (Figure 2);

3. $\arg R^{n} \longrightarrow 2 k \pi / p$ locally uniformly on $\Pi_{k}(t)$ as $n \longrightarrow \infty$ (Figure 2);

4. $|R(z)|<|z|$ on a neighborhood of the axis of each petal (Figure 2); 
5. $R: \Pi_{k}(t) \longrightarrow \Pi_{k}(t)$ is conjugate to a translation.

[Beardon, p. 116]

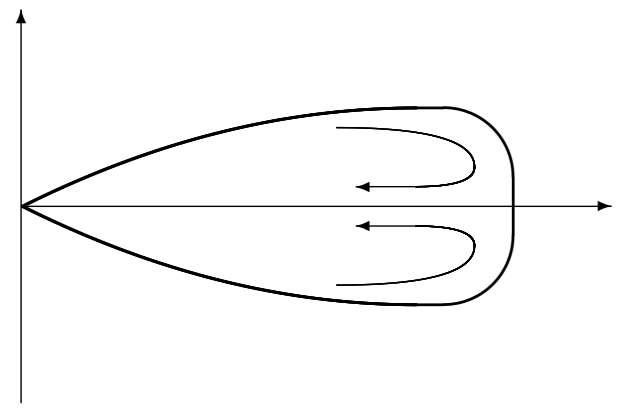

Figure 2

For an arbitrary rational function $R$ with a rationally indifferent fixed point at 0 , whose multiplier is equal to 1 , we have

Lemma 1 Let $R$ be as above with a Taylor expantion

$$
R(z)=z+a z^{p+1}+O\left(z^{p+2}\right), \quad a \neq 0
$$

at 0 . Then $R$ is conjugate near 0 to a function of the form

$$
R(z)=z-z^{p+1}+O\left(z^{2 p+1}\right) .
$$

[Beardon, p. 122-124] 
Therefore the petals of such function are the conformal images of $\Pi_{k}(t)$ under some conjugating map. The conclusions of the Petal Theorem are valid for this arbitrary rational function except for the fact that we no longer have an explicit expression for the petals, however each deformed petal still subtends an angle $2 \pi / p$ at the origin, and the (small enough) petals are pairwise disjoint (since the conjugacies are all conformal maps).

If $R$ has the following Taylor espansion at the origin:

$$
R(z)=a z+b z^{p+1}+\cdots
$$

where $a \neq 1$ but $a=\exp (2 \pi i r / q)$ for positive integers $r, q$ which are coprime, then $R^{q}$ has the form

$$
R^{q}(z)=z+c z^{l+1}+\cdots
$$

for some positive integer $l$, so $R^{q}$ has $l$ petals at the origin, which yields that $R$ is a composition of, say, $k$ disjoint cycles each of length $q$, such that $l=k q$. Thus in this case, $R$ has $l=k q$ petals at the origin, these dividing into $k$ sets of $q$ petals such that $R$ acts as a cycle of length $q$ on each such set. The positive integer $l$ is equal to $p$ if and only if the numbers $p$ and $q$ are coprimes. [Beardon, p. 130]

Now let the point 0 belong to a rationally indifferent cycle $\left\{\zeta_{0}, \cdots, \zeta_{m-1}\right\}$ of $R$, say $\zeta_{0}=0$, and $\zeta_{i}=R^{i}(0)$. The essential difference between this case and the previous one is that now, $R$ maps each connected component of its Fatou set that contains a petal at $\zeta_{j}$ to some other component which contains a petal at $\zeta_{j+1}$, where $j=0, \cdots, m-1$. Since $R^{m}$ fixes each $\zeta_{j}$ in the cycle, the previous version applies to $R^{m}$. Indeed, we have the following

Theorem 16 (The General Theorem of Petals) Let $\left\{\zeta_{0}, \cdots, \zeta_{m-1}\right\}$ be a rationally indifferent cycle for $R$, and let the multiplier of $R^{m}$ at each 
point of the cycle be $\exp (2 \pi i r / q)$, where $(r, q)=1$ (they are coprimes). Then there exist an integer $l$, and mlq distinct components $F_{0}, \cdots, F_{m l q-1}$ of the Fatou set $\mathbf{F}(R)$ such that at each $\zeta_{j}$, there are exactly $l q$ of these components containing a petal of angle $2 \pi / l q$ at $\zeta_{j}$. Further, $R$ acts as a permutation $\tau$ on $\left\{F_{0}, \cdots, F_{m l q-1}\right\}$, where $\tau$ is a composition of $l$ disjoint cycles of length $m q$, and a petal based at $\zeta_{j}$ maps under $R$ to a petal based at $\zeta_{j+1}$. Beardon, p. 131]

There are some other points in $\overline{\mathbf{C}}$ which play an important role in determining the behavior of the family $\Upsilon$ on $\overline{\mathbf{C}}$, namely the critical points of $R$.

Definition 14 The point $z_{0} \in \overline{\mathbf{C}}$ is a critical point of a rational map $R$ if $R$ is not injective in any neighborhood of $z_{0}$. The orbit of such a point is called a critical orbit.

The two main theorems concerning critical points, which we will need later are

Theorem 17 (Riemann-Hurewitz) For every non-constant rational map $R$ of degree $d$, the number of the critical points of $R$ counted with multiplicity is at most 2d-2. [Beardon, p. 43]

Theorem 18 Let $C$ be the set of all critical points of the rational map $R$. Then the set of all critical values of $R^{n}$ is

$$
R(C) \cup \cdots \cup R^{n}(C) .
$$

[Beardon, p. 45]

Definition 15 The postcritical set of $R$ is 


$$
C^{+}=\bigcup_{n=0}^{\infty} R^{n}(C),
$$

where $C$ is the set of critical points of $R$.

Theorem 19 Let $R$ be a rational map of degree at least 2 , and $\Upsilon=\left\{R^{n}\right\}_{n \in \mathbf{N}}$. Then

1. The immediate basin of each (super-)attracting cycle of $\Upsilon$ contains a critical point of $R$;

2. Each immediate basin of a rationally indifferent cycle of $\Upsilon$ has a critical point of $R$.

3. Let $\left\{\Omega_{1}, \cdots, \Omega_{q}\right\}$ be a cycle of Siegel discs or Herman rings of $\Upsilon$. Then $\cup \partial \Omega_{j} \subset \overline{C^{+}}$.

4. Every irrationally indifferent cycle of $\Upsilon$ which belongs to $\mathbf{J}(\Upsilon)$, lies in the derived set of $C^{+}$.

[Beardon, chap. 9]

Definition 16 A rational map $R$ is called hyperbolic if $R$ is expanding on the Julia set $\mathbf{J}(\Upsilon)$, i.e. there is a Riemannian metric $\|$.$\| , defined on a$ neighborhood of $\mathbf{J}(\Upsilon)$, such that the derivative $D R_{z}$ at every point $z \in \mathbf{J}(\Upsilon)$ satisfies

$$
\left\|D R_{z}(v)\right\|>\|v\|
$$

for every nonzero vector $v$ in the tangent space $T \overline{\mathbf{C}}_{z}$. 
Since $\mathbf{J}(\Upsilon)$ is a compact set, there exists some $k>1$ with the property $\left\|D R_{z}(v)\right\|>k\|v\|$ for all points $z$ in some neighborhood of $\mathbf{J}(\Upsilon)$, and for every nonzero vector $v$ in the tangent space $T \overline{\mathbf{C}}_{z}$. Furthermore if $R$ is hyperbolic with respect to one Riemannian metric, it is hyperbolic with respect to every Riemannian metric Carleson-Gamelin.

Theorem 20 A rational map $R$ of degree at least 2 is hyperbolic if and only if the closure of its postcritical set is disjoint from the Julia set $\mathbf{J}(\Upsilon)$ related to it. [Milnor, p. 189]

Or more:

Theorem 21 A rational map $R$ of degree at least 2 is hyperbolic if and only if the orbit of every critical point of $R$ converges to a (super-) attracting cycle. [Milnor, $p$. 189]

According to Mañe, Sad, Sullivan, the Julia set $\mathbf{J}(\Upsilon)$ related to the hyperbolic map $R$ deforms continuously under deformations of $R$ through hyperbolic maps. Here we have another important

Theorem 22 If the Julia set of a hyperbolic map is connected, then it is locally connected. [Milnor, p. 191]

One can consider a wider class of rational functions, namely of those which have critical points in their Julia sets only if each orbit of such a critical point is allowed to have only finitely many elements. Such rational functions are called subhyperbolic. Obviously, the class of subhyperbolic rational functions 
contains the hyperbolic ones. In order to define subhyperbolicity exactly, we need some additional definitions. The first two of them are rather topological. We recall that a map $f: X \longrightarrow Y$ from the topological space $X$ to the topological space $Y$ is called a proper map if the inverse image $f^{-1}(K)$ of each compact subset $K$ of $Y$ is a compact subset of $X$.

Definition 17 A holomorphic map $p: S^{\prime} \longrightarrow S$ between two Riemann surfaces will be called a branched covering map if every point of $S$ has a connected neighborhood $U$ so that each connected component of $p^{-1}(U)$ maps onto $U$ by a proper map. [Milnor]

Definition 18 Let $f: D \longrightarrow \overline{\mathbf{C}}$ be a holomorphic function defined from a domain $D \subset \overline{\mathbf{C}}$ into the Riemann's sphere so that in a suitable neighborhood of a point $z_{0} \in D$ :

$$
f(z)=w_{0}+c\left(z-z_{0}\right)^{n}+(\text { higher terms }) .
$$

The integer $n\left(f, z_{0}\right)=n \geq 1$ is called the local degree of $f$ at the point $z_{0}$. Hence $n(z) \geq 2$ if $z$ is a critical point, and $n(z)=1$ otherwise.

Definition 19 A metric on a domain $D \subset \overline{\mathbf{C}}$ with the expression $\gamma(z)|d z|$ is called an orbifold metric if the function $\gamma(z)$ is smooth and nonzero on $D$ except at a locally finite collection of points $a_{1}, a_{2}, \cdots$ known as ramification points. [Milnor]

\section{Definition 20}

- The singularities $a_{1}, a_{2}, \cdots$ of such an orbifold metric are points for which some integers known as ramification indices $\nu_{j} \geq 2$ at $a_{j}$ are defined so that if one takes a local branched covering by setting $z=\phi_{a_{j}}(w)=a_{j}+w^{\nu_{j}}$, then the induced metric $\gamma(z(w))\left|\frac{d z}{d w}\right| \cdot|d w|$ on the w-plane is smooth and nonzero throughout some neighborhood of the origin. 
- An orbifold $(S, \nu)$ is a subspace $S \subset \overline{\mathbf{C}}$, together with a localy finite collection of marked points $a_{j}$ which are called ramified points, each of which is assigned a ramification index $\nu_{j} \geq 2$ as above. For any point $z$ which is not one of the $a_{j}$ we set $\nu(z)=1$. [Milnor, $p$. 196]

Definition 21 The rational function $R$ is expanding with respect to an orbifold metric $\gamma(z)|d z|$ if the absolute value $\|$.$\| of its derivative in this metric$ satisfies

$$
\left\|D R_{z}\right\| \geq k>1
$$

whenever $z$ and $R(z)$ are not one of the above $a_{j}$ 's. In other words:

$$
\gamma(R(z))\left|R^{\prime}(z)\right| \geq k \gamma(z)
$$

where |.| is the spherical metric, and $z$ and $k$ are as above.

Definition 22 The rational map $R$ is subhyperbolic if it is expanding with respect to some orbifold metric on a neighborhood of its Julia set. [Milnor, p. 195]

If a rational function $R$ is subhyperbolic, and $c$ is a critical point in its Julia set, then every forward image $R^{n}(c), n>0$, must be one of the ramification points $a_{1}, \cdots, a_{q}$ of the orbifold metric in the Julia set, since the function $R^{n}$ has derivative zero at the critical point $c$, and yet must satisfy $\left\|D R_{z}^{n}\right\| \geq k^{n}$ at the points arbitrarily close to $c$ ([Milnor $]$ ). Indeed, we have the following

Theorem 23 A rational map is subhyperbolic if and only if each of its critical orbits is either finite or converges to an attracting cycle. [Milnor, $p$. 195] 
The proof of this theorem contains the construction of an orbifold metric for a given rational function with the critical orbits as mentioned in the theorem. To each such function one assigns the canonical orbifold $(S, \nu)$ as follows. As the underlying surface $S$ we take the Riemann Sphere $\overline{\mathbf{C}}$ with all (super)attracting periodic orbits removed. As ramification points $a_{j}$ we take all strictly postcritical points $R^{n}(c)$, where $c$ is a critical point and $n>0$. In order to specify the ramification indices $\nu\left(a_{j}\right)$ we consider all pairs $(c, m)$ where $\mathrm{c}$ is a critical point with $R^{m}(c)=a_{j}$, and choose $\nu\left(a_{j}\right)=\nu_{j}$ to be the least common multiple of the corresponding local degrees $n\left(R^{m}, c\right)($ $a_{j}$ itself may be a critical point, since one critical point may eveutualy map to another). There are only finitely many such pairs $(c, m)$ since we have removed all superattracting periodic orbits, so this least common multiple is well defined and finite. If $R(z)$ is an attracting periodic point, and hence not in $S$, then we set $\nu(R(z))=\infty$. For any other $z \in S \backslash\left\{a_{1}, a_{2}, \cdots\right\}$ the ramification index $\nu(z)$ is defined to be 1 . So we can define the orbifold metric $\gamma(z)|d z|$ as before, i.e. $\gamma(z)$ is smooth and nonzero except at ramification points $a_{1}, a_{2}, \cdots$, and if we take a local branched covering at the point $a_{j}$ by setting $z=\phi_{j}(w)=a_{j}+w^{\nu_{j}}$, then the induced metric $\gamma(z(w))\left|\frac{d z}{d w}\right| \cdot|d w|$ on the $w$-plane is smooth and nonzero throughout some neighborhood of the origin. Milnor

We illustrate these ideas in the following

Example. Consider $P(z)=z^{2}-2$. The critical points are 0 and $\infty$. The point $\infty$ is indeed a superattracting fixed point. The postcritical points are $\infty \longrightarrow \infty$ and $0 \longrightarrow-2 \longrightarrow 2$. The surface $S$ is defined to be the complex plane $\mathbf{C}=\overline{\mathbf{C}} \backslash\{\infty\}$. The Julia set of the family $\left\{P^{n}\right\}_{n \in \mathbf{N}}$ is the closed set $[-2,2]$ Carleson-Gamelin, p. 29]. The function $\mathrm{P}$ is not hyperbolic, since the postcritical set is in the Julia set. But $P$ is subhyperbolic, since the set 
of the postcritical points is finite. The ramification points are $a_{1}=-2$ and $a_{2}=2$ both in the Julia set $\mathcal{J}(P)$. The first ramification index $\nu\left(a_{1}\right)$ is the local degree of $P$ at 0 , hence $\nu\left(a_{1}\right)=2$. The second one $\nu\left(a_{2}\right)$ is the local degree of $P^{2}$ at 0 . The local form of $P^{2}$ at 0 is

$$
P(z)=2-4 z^{2}+z^{4}
$$

Therefore $\nu\left(a_{2}\right)=2$, too.

The canonical orbifold metric $\gamma(z)|d z|$ must have singularities at the ramification points. We define this metric by

$$
\gamma(z):=\frac{1}{\sqrt{|z+2||z-2|}}
$$

In a neighborhood $U_{1}$ of $a_{1}$ we set $z=\phi_{1}(w)=-2+w^{2}$. This is a local branched covering map from $U_{1}$ to a neighborhood of the origin. Then the induced metric $\gamma\left(\phi_{1}(w)\right)\left|\frac{d \phi_{1}}{d w}\right| .|d w|$ has the form $\frac{1}{\sqrt{\left|w^{2}-4\right|}}|d w|$ which is smooth and nonzero at the origin.

Similarly, we choose $z=\phi_{2}(w)=2+w^{2}$ in a neighborhood $U_{2}$ of $a_{2}$ which produces a local branched covering map from $U_{2}$ to some neighborhood of the origin. The induced metric $\gamma\left(\phi_{2}(w)\right)\left|\frac{d \phi_{2}}{d w}\right| .|d w|$ is then equal to $\frac{1}{\sqrt{\left|w^{2}+4\right|}}|d w|$ which is again smooth and nonzero at the origin.

The next step is to show that $\gamma(P(z))\left|P^{\prime}(z)\right| \geq k \gamma(z)$, which should mean that $P(z)$ is expanding with respect to the orbifold metric $\gamma(z)|d z|$. For $k=2$ we have the equality $\gamma(P(z))\left|P^{\prime}(z)\right|=k \gamma(z)$ on $\mathbf{C} \backslash\left\{a_{1}, a_{2}\right\}=\mathbf{C} \backslash\{-2,2\}$ and therefore on a neighborhood of the Julia set $[-2,2]$, which was our purpose.

There is another theorem concerning subhyperbolic functions which will be used in the next chapter. 
Theorem 24 If $R$ is subhyperbolic, and its Julia set is connected, then the Julia set is locally connected. [Milnor, p. 195]

Denote the Lebesgue measure generated by the spherical metric on the Riemann Sphere $\overline{\mathbf{C}}$ by $\mu$ (sec. 2.1.1, p. 7).

Definition $23 A$ set $Y$ is said to be a wandering set if $R^{-m}(Y) \cap Y=\emptyset$ for every positive integer $m \geq 1$. A point $z$ is called a wandering point if it has a wandering open neighborhood. The set of wandering points on $\overline{\mathbf{C}}$ is denoted by $\mathcal{B}$, and its complement is denoted by $\Omega$ Lyubich 1983.

The above definition works if we consider the condition $R^{m}(Y) \cap R^{n}(Y)=$ $\emptyset$ for every two nonegative integers $m \neq n$ instead of $R^{-m}(Y) \cap Y=\emptyset$ for every positive integer $m \geq 1$, since these two conditions are equivalent. From Theorem 10 in this section we see that $\mathbf{J}(\Upsilon) \subset \Omega$, where $\Upsilon=\left\{R^{n}\right\}_{n \in \mathbf{N}}$ as before.

Theorem 25 Let $\Omega \neq \overline{\mathbf{C}}$. If the rational function $R$ is subhyperbolic, then the orbits of almost all points on $\overline{\mathbf{C}}$ (with respect to the measure $\mu$ ) converge to (super-)attracting cycles. Consequently, $\mu(\Omega)=0$ in this case. Eremenko-Lyubich

Hence if $R$ is subhyperbolic, then $\mu(\mathbf{J}(\Upsilon))=0$. Indeed, in this case the set $\Omega \backslash \mathbf{J}(\Upsilon)$ consists only of (super-)attracting periodic points. This, however, does not happen in the general case, i. e. when the function $R$ is not subhyperbolic, although the Julia set of the family of the iterates of $R$ is always nowhere dense. See for example Lyubich 1986. 


\subsection{Random Iterations: General Case}

Let $\left\{f_{n}\right\}_{n \in \mathbf{N}}$ be an arbitrary family of rational maps of degree at least 2 on the Riemann's sphere. What we will study is the family $\mathcal{F}=\left\{F_{n}\right\}_{n \in \mathbf{N}}$, where $F_{n}=f_{n} \circ \cdots \circ f_{1}$.

This family is an extension of the standard case of iterations of one rational map mentioned before. So the natural question is how far we can extend the results of the classical iteration theory to such families. The general case, where there are no restrictions on $f_{j}$ 's, has been studied by M. Bueger in Bueger. We mention here some results from Bueger and other references to provide a suitable background for the next sections.

Theorem 26 The Julia set of the family $\mathcal{F}$ is nonempty. Bueger

\section{Proof.}

We prove by contradiction. Let the Julia set $\mathbf{J}$ be an empty set. Then there is a subsequence $\left\{F_{n_{k}}\right\}$ of $\left\{F_{n}\right\}$ and a function $G$ such that

$$
\lim _{k \rightarrow \infty} F_{n_{k}}=G \text {, locally uniformly on } \overline{\mathbf{C}} \text {. }
$$

Indeed this subsequence is uniformly convergent on the whole $\overline{\mathbf{C}}$, since $\overline{\mathbf{C}}$ is a compact space. Therefore the function $G$ must be meromorphic on $\overline{\mathbf{C}}$ and hence rational. There are two cases to be considered:

- The function $G$ is a constant function.

We can assume $G$ to be identically equal to $\infty$, since otherwise we can map it to $\infty$ by a translation. But then 


$$
\lim _{k \rightarrow \infty} F_{n_{k}}=\infty
$$

i. e. for every $\epsilon>0$ there is some positive integer $k_{0}$ such that for each $k>k_{0}, \chi\left(F_{n_{k}}(z), \infty\right) \leq \epsilon$. As we have mentioned before, $F_{n_{k}}$ is not constant and has some zero $z_{k}$. Therefore for every positive integer $\mathrm{k}$ there is some complex number $z_{k}$ such that $\chi\left(F_{n_{k}}\left(z_{k}\right), \infty\right)=\chi(0, \infty)=$ 2, a contradiction.

- The limit function $G$ is not constant.

The number $\mathrm{N}$ of the zeros of $G$ counted with multiplicity is an integer between 1 and the degree of $G$ : $1 \leq N \leq \operatorname{deg} G$. We can find some disjoint open discs $U_{1}, \cdots, U_{N}$ at the zeros of $G$ such that $\cup_{k=1}^{N} U_{k}$ has no poles of the function $G$. Denote the compact complement of $\cup_{k=1}^{N} U_{k}$ in $\overline{\mathbf{C}}$ by $\mathrm{K}$ and $\min \{|G(z)|: z \in K\}$ by $\epsilon$. Therefore $\epsilon>0$. From the uniform convergence of $F_{n_{k}}$ to $G$ on $\mathrm{K}$ we can find some $k_{0}$ such that for every $k>k_{0}$ and each $z \in K$

$$
\chi\left(F_{n_{k}}(z), G(z)\right)=\left|F_{n_{k}}(z)-G(z)\right| \leq \epsilon \leq|G(z)| .
$$

Hence from Rouché Theorem $F_{n_{k}} \neq 0$. Further we deduce that $\mathrm{G}$ and $F_{n_{k}}$ have the same number of zeros (with multiplicity) on $\cup_{k=1}^{N} U_{k}$ and therefore on $\overline{\mathbf{C}}$ for $k \geq k_{0}$, i. e. for such k's $\operatorname{deg} G=\operatorname{deg} F_{n_{k}}$. But $f_{n_{k}}$ 's are all rational functions with $\operatorname{deg} f_{n_{k}} \geq 2$. Hence $\operatorname{deg} F_{n_{k}}$ goes to $\infty$ as $k \longrightarrow \infty$, a contradiction.

Theorem 27 For each positive integer n:

1. $F_{n}^{-1}\left(F_{n}(\mathbf{F})\right) \subset \mathbf{F}$;

2. $F_{n}^{-1}\left(F_{n}(\mathbf{J})\right) \subset \mathbf{J}$. 


\section{Bueger}

\section{Proof.}

1. Let $\mathrm{z}$ be in $\mathbf{F}$, there is a neighborhood $\mathrm{U}$ of $\mathrm{z}$ where $\left\{F_{n_{k}}\right\}$ is defined and normal. Denote $U_{n}:=F_{n}(U)$, and $G_{k}:=f_{n+k} \circ \cdots \circ f_{n+1}$, for positive integers k. Thus the family $\left\{G_{n}\right\}$ is defined and normal in $U_{n}$. For $w \in F_{n}^{-1}\left(F_{n}(z)\right)$ there exists a neighborhood $\mathrm{V}$ with the property $F_{n}(V) \subset U_{n}$. Hence the family $\left\{G_{n}\right\}$ is normal in $U_{n}$ from which we deduce that $\left\{F_{k}\right\}$ is normal in $\mathrm{V}$, i. e. $w \in \mathbf{F}$.

2. Now assume $z \in \mathbf{J}$ and $w \in F_{n}^{-1}\left(F_{n}(z)\right)$. Thus $F_{n}(w)=F_{n}(z)$ which means $z \in F_{n}^{-1}\left(F_{n}(w)\right)$. Therefore if $w \in \mathbf{F}$, then from the first part we have $z \in \mathbf{F}$, a contradiction.

For an arbitrary family $\mathcal{F}$, the Julia set $\mathbf{J}(\mathcal{F})=\mathbf{J}$ is not necessarily nowhere dense. But under some conditions on the sequence $\left\{f_{n}\right\}$ we have this property again:

Theorem 28 Let $U \subset \overline{\mathbf{C}}$ be a nonempty open set whose complement has at least three points. If the sequence $\left\{f_{n}\right\}_{n \in \mathbf{N}}$ satisfies $f_{n}(U) \subset U, \forall n \in \mathbf{N}$, then $U \subset \mathbf{F}$, and $\mathbf{J}$ is nowhere dense. $U$ is called an invariant domain of the family $\mathcal{F}$. Bueger

\section{Proof.}

For every positive integer $\mathrm{n}$ we have $f_{n}(U) \subset U$. Hence $F_{n}(U) \subset U$. Since $\sharp(\overline{\mathbf{C}} \backslash U) \geq 3$, we deduce from Montel's Theorem that $\left\{F_{n}\right\}$ is normal in U, which means $U \subset \mathbf{F}$.

Now we show by contradiction that $\mathbf{J}$ is nowhere dense: otherwise there is some domain $D \subset \mathbf{J}$ such that $\left\{F_{n}\right\}$ is holomorphic in D. 
We claim there is some positive integer $\mathrm{n}$ such that $F_{n}(D) \cap U \neq \emptyset$; otherwise from Montel's theorem $D \subset \mathbf{F}$ (as $\mathrm{U}$ is open and not empty), which is a contradiction.

Hence there is a domain $V \subset D$ with $F_{k}(V) \subset U$, and therefore $F_{n}(V) \subset$ $U$ for every $n \geq k$. According to Montel's Theorem it can not occure, since then $V \subset \mathbf{F}$. This completes the proof.

Let $\mathrm{V}$ be a stable domain (cf. Definition 7, sec. 2.1.2) of $\mathbf{F}$ and $\mathrm{W}$ be a connected component of $F_{n}^{-1}\left(F_{n}(V)\right)$, then $W \subset \mathbf{F}$. Indeed, we have $\partial W \subset \mathbf{J}$ Bueger, p. 41]: if there is some $z_{0}$ in $\partial W \cap \mathbf{F}$, then there is some connected neighborhood $U$ of $z_{0}$ such that $U \subset \mathbf{F}$. Specially, $U \cap W \neq \emptyset$. Therefore $F_{n}(U) \cap F_{n}(W) \neq \emptyset$ which means $W \cap F_{n}^{-1}\left(F_{n}(U)\right) \neq \emptyset$. Let U' be a connected component of $F_{n}^{-1}\left(F_{n}(U)\right)$ which meets $\mathrm{W}$. The case $U^{\prime} \subset W$ can not accure, since then $F_{n}(U)=F_{n}\left(U^{\prime}\right) \subset F_{n}(W)$ which yields $U \subset W$. Therefore $U^{\prime} \cap \partial W \neq \emptyset$, and $U^{\prime} \cap \mathbf{J} \neq \emptyset$ which contradicts Theorem 27. Thus we have

Theorem 29 Let $V$ be a stable domain. Then for every positive integer $n$, every connected component of $F_{n}^{-1}\left(F_{n}(V)\right)$ is also a stable domain. Bueger

An easy application of Theorem 27 and Theorem 29 yields

Theorem 30 Let $V$ and $W$ be two stable domains of the family $\mathcal{F}$. Then either $F_{n}(V)=F_{n}(W)$ or $F_{n}(V) \cap F_{n}(W)=\emptyset$ for every positive integer n. Bueger, p. 41]

Theorem 31 Let $V$ be a stable domain. Then the following two statements are equivalent

1. $V$ is a contracting domain; 
2. For every $z, w$ in $V$,

$$
\chi\left(F_{n}(z), F_{n}(w)\right) \longrightarrow 0 \quad \text { as } \quad n \longrightarrow \infty
$$

Bueger

Proof.

First we show that $1 \Rightarrow 2$. Let $\mathrm{V}$ be contracting domain and $z, w \in V$ such that $\chi\left(F_{n}(z), F_{n}(w)\right) \nrightarrow 0$ as $n \longrightarrow \infty$. Hence there are a subsequence $\left(n_{k}\right)$ and some positive $\epsilon$ for which we have $\chi\left(F_{n_{k}}(z), F_{n_{k}}(w)\right) \geq \epsilon$ for every positive integer k. As $\left\{F_{n}\right\}$ is normal in $\mathrm{V}$, it has a subsequence $\left\{F_{n_{k}}\right\}$ convergent in $\mathrm{V}$. We denote its limit function by $\mathrm{F}$. But $\mathrm{V}$ is a contracting domain (cf. Definition 8, sec. 2.1.2), i. e. $\mathrm{F}$ is a constant function: $F \equiv c$ for some complex number $c \in \overline{\mathbf{C}}$. We conclude

$$
F_{n_{k}}(z) \longrightarrow c \text { and } F_{n_{k}}(w) \longrightarrow c \text { as } k \longrightarrow \infty
$$

which is a contradiction.

Now we will show that $2 \Rightarrow 1$. Let $\mathrm{F}$ be in $\mathcal{G}(V)$ and $\left\{F_{n_{k}}\right\}$ be a subsequence of $\left\{F_{n}\right\}$ convergent to $\mathrm{F}$ on $\mathrm{V}$. For some arbitrary $w \in V$ we put $F(w)=c$. Then for every $\mathrm{z}$ in $\mathrm{V}$ :

$$
\chi\left(F_{n_{k}}(z), c\right) \leq \chi\left(F_{n_{k}}(z), F_{n_{k}}(w)\right)+\chi\left(F_{n_{k}}(w), c\right) .
$$

The first summand on the right goes to 0 according to the assumption 2 . As $F(w)=c$, the second summand goes to 0 , too. Hence for every $\mathrm{z}$ in $\mathrm{V}$

$$
\left(F_{n_{k}}(z), F_{n_{k}}(w)\right) \longrightarrow 0 \text { as } k \longrightarrow 0
$$

which means $F \equiv c$ for some $c \in \overline{\mathbf{C}}$.

Similar to the standard case we can define here the notion of a periodic point of the family $\mathcal{F}$ : 
Definition 24 If there exist some $z_{0} \in \overline{\mathbf{C}}$ and some positive integer $n_{0}$ such that for every integer $n \geq n_{0}, f_{n}\left(z_{0}\right)$ is defined and equal to $z_{0}$, then $z_{0}$ is called a periodic point of order $n_{0}$ of the family $\mathcal{F}$. Bueger]

Theorem 32 Let $z_{0}$ be a periodic point of order $n_{0}$. If the sequence $\left\{\prod_{n}\left|f_{n}^{\prime}\left(z_{0}\right)\right|\right\}_{n \geq n_{0}}$ is divergent, then $F_{n_{0}}^{-1}\left(z_{0}\right) \subset \mathbf{J}$. We call such $z_{0}$ a repelling periodic point of order $n_{0}$ of the family $\mathcal{F}$. Bueger, p.23] 


\section{The Subhyperbolic Relaxed Newton's Method}

\subsection{Introductory Remarks}

Let $P_{0}(z) \in \mathcal{R}_{d}$ be a polynomial of the form

$$
P_{0}(z)=a_{0}+a_{1} z+\cdots+a_{d} z^{d}, \quad d \geq 2 \text { and } a_{d} \neq 0
$$

with $\left[\left(a_{0}, \cdots, a_{d}\right):(1, \cdots, 1)\right] \in \mathbf{C P}^{d}$.

The relaxed Newton's method for such a polynomial is by definition

$$
(*) \quad N_{P_{0}, h_{0}}(z)=z-h_{0} \frac{P_{0}(z)}{P_{0}^{\prime}(z)},
$$

where $h_{0}$ is an arbitrary number in $(0,1]$.

Therefore,

$$
N_{P_{0}, h_{0}}^{\prime}(z)=1-h_{0}+h_{0} \frac{P_{0}(z) P_{0}^{\prime \prime}(z)}{\left(P_{0}^{\prime}(z)\right)^{2}}
$$

If $h_{0}=1$, the formula $(*)$ takes the form

$$
N_{P_{0}}(z)=z-\frac{P_{0}(z)}{P_{0}^{\prime}(z)}
$$

which is called the Newton's method for the polynomial $P_{0}$. In this case $z_{0}$ is a simple root of $P_{0}$ if and only if $z_{0}$ is a superattracting fixed point of the rational function $N_{P_{0}}$.

From now on we assume the following conditions: 
1. $P_{0}$ has at least two different roots;

2. $N_{P_{0}, h_{0}}$ is a subhyperbolic rational function.

These facts are then immediate:

- $N_{P_{0}, h_{0}}$ is a rational function of degree $d$ with $2 \leq d \leq \operatorname{deg}\left(P_{0}\right)$.

- $z_{0} \in \overline{\mathbf{C}}$ is a zero of $P_{0}$ of multiplicity $k$ if and only if $z_{0}$ is an attracting fixed point of $N_{P_{0}, h_{0}}$ of multiplicity $\frac{k-h}{k}$.

- If $N_{P_{0}, h_{0}}^{\prime}(c)=0$ for some $c \in \overline{\mathbf{C}}$, then the set $\left\{N_{P_{0}, h_{0}}^{n}(c)\right\}_{n \in \mathbf{N}}$ has either finitely many elements, or every accumulation point of it is a (super-) attracting periodic point of the family $\left\{N_{P_{0}, h_{0}}^{n}\right\}_{n \in \mathbf{N}}$.

- If for such $c$ as in the last paragraph $\left\{N_{P_{0}, h_{0}}^{n}(c)\right\}$ converges to some complex number $z_{0}$, then

$$
N_{P_{0}, h_{0}}\left(z_{0}\right)=N_{P_{0}, h_{0}}\left(\lim _{n \rightarrow \infty} N_{P_{0}, h_{0}}^{n}(c)\right)=\lim _{n \rightarrow \infty} N_{P_{0}, h_{0}}^{n+1}(c)=z_{0} ;
$$

thus if for every critical point $c$ of $N_{P_{0}, h_{0}}$, the orbit of $c$ converges, then the limit point is an attracting fixed point, which means there are no attracting periodic points of period $\geq 2$. Hence the Fatou set $\mathbf{F}\left(N_{P_{0}, h_{0}}\right)$ is equal to $\bigcup_{P_{0}(\zeta)=0} \mathcal{A}(\zeta)$, where $\mathcal{A}(\zeta)$ is the basin of attraction of $\zeta$.

- $\infty$ is a repelling fixed point of $N_{P_{0}, h_{0}}$ with multiplier $\frac{d}{d-h_{0}}$.

Indeed, $\infty$ is the only repelling fixed point for the family $\left\{N_{P_{0}, h_{0}}^{n}\right\}_{n \in \mathbf{N}}$. From a theorem due to Shishikura Shishikura, Corollary II of Theorem I] we deduce that the Julia set $\mathbf{J}\left(N_{P_{0}, h_{0}}\right)$ is connected, therefore also locally connected, since $N_{P_{0}, h_{0}}$ is subhyperbolic ([Milnor, chap. 19]). 
Now let $\left\{P_{n}\right\}_{n \in \mathbf{N}}$ be a sequence of polynomials

$$
P_{n}(z)=a_{n, 0}+a_{n, 1} z+\cdots+a_{n, d} z^{d}
$$

all in $\mathcal{R}_{d}$ with $a_{n, d} \neq 0$, and $\left\{h_{n}\right\} \subset \subset(0,1]$ be a sequence of real numbers. These two families produce a sequence of relaxed Newton's methods $\left\{N_{P_{n}, h_{n}}\right\}_{n \in \mathbf{N}}$ which works as the base family for the main sequence $\left\{N_{n}:=N_{P_{n}, h_{n}} \circ \cdots \circ N_{P_{1}, h_{1}}\right\}$, i. e. we are now in the zone of random iterations of rational functions. According to the different conditions on the sequence $\left\{P_{n}\right\}_{n \in \mathbf{N}}$ we have different dynamical behaviors for the family $\left\{N_{n}\right\}$, which will be studied in following sections.

We denote the open disc of radius $\mathrm{r}$ and center $\mathrm{z} \in \overline{\mathbf{C}}$ (in the relevant topology) by $D_{r}(z)$. 


\subsection{Near $\left(\mathbf{P}_{0}, \mathbf{h}_{0}\right)$}

\subsubsection{Introduction}

Let the real number $\varepsilon>0$ be so small that $U_{\varepsilon}\left(P_{0}\right)$ lies in $\mathcal{R}_{d}$ (each $\mathcal{R}_{d}$ is an open set, and $d \geq 2$ ), and $h_{0}>0 . U_{\varepsilon}\left(P_{0}\right)$ is as mentioned before the $\varepsilon$-neighborhood of $P_{0}$ in $\mathcal{R}_{d}$ with respect to the uniform metric.

Beginning with a sequence of polynomials $\left\{P_{n}\right\}_{n \in \mathbf{N}}$ in $U_{\varepsilon}\left(P_{0}\right)$ and a sequence of real numbers $\left\{h_{n}\right\}$ in the interval $\left(\max \left\{0, h_{0}-\varepsilon\right\}, \min \left\{1, h_{0}+\varepsilon\right\}\right]$ of $h_{0}>0$, we consider the family $\left\{N_{n}\right\}_{n \in \mathbf{N}}$ where $N_{n}=N_{P_{n}, h_{n}} \circ \cdots \circ N_{P_{1}, h_{1}}$. Our aim is to find out how small this $\varepsilon$ can be chosen to let the family $\left\{N_{n}\right\}$ have a dynamical behavior similar to that of the standard family $\left\{N_{P_{0}, h_{0}}^{n}\right\}_{n \in \mathbf{N}}$.

As $N_{P_{n}, h_{n}}$ 's are nonconstant rational functions of degree at least 2 , we note that $\left\{N_{n}\right\}$ is a family of rational functions of degree at least 2 .

\subsubsection{The Julia Set}

The inverse function $\phi(z)=\frac{1}{z}$ maps a small neighborhood $U$ of 0 to a neighborhood of $\infty$ so that the map $M_{P_{0}, h_{0}}=\phi^{-1} \circ N_{P_{0}, h_{0}} \circ \phi$ has the form

$$
M_{P_{0}, h_{0}}(z)=\alpha_{0} z+\cdots, \quad\left|\alpha_{0}\right|>1
$$


in $U$. The maps $N_{P_{n}, h_{n}}$ 's are the relaxed Newton's methods for $P_{n}$ 's and $h_{n}$ 's.

Since each component $\mathcal{R}_{d}$ is open in $\mathcal{R}$, we can choose $\varepsilon$ so that for every polynomial $P_{n}$ in the neighborhood $U_{\varepsilon}\left(P_{0}\right) \subset \subset \mathcal{R}_{d}$ of $P_{0}$, and every $h_{n}$ in the real interval $\left(\max \left\{0, h_{0}-\varepsilon\right\}, \min \left\{1, h_{0}+\varepsilon\right\}\right]$ around $h_{0}$ the maps $M_{P_{n}, h_{n}}(z)=\phi^{-1} \circ N_{P_{n}, h_{n}} \circ \phi(z)$ have the form

$$
M_{P_{n}, h_{n}}(z)=\alpha_{n} z+(\text { terms of higher degrees of } \mathrm{z})
$$

and for some $C>1$

$$
\inf _{n}\left|\alpha_{n}\right| \geq C, \quad \text { in } U
$$

We denote this modified $\varepsilon$ by $\varepsilon_{0}$.

Theorem 1 The Julia set $\mathbf{J}$ of $\left\{N_{n}\right\}$ is nonempty.

\section{Proof.}

The point $\infty$ is a repelling fixed point (of order 1) for the family $\left\{N_{n}\right\}$, or 0 is a repelling fixed point for the family $\left\{M_{n}=M_{P_{n}, h_{n}} \circ \cdots \circ M_{P_{1}, h_{1}}\right\}$. Therefore 0 is in the Julia set of the family $\left\{M_{n}\right\}$, since otherwise $\left\{M_{n}\right\}$ is normal at $0, \mathrm{i}$. e. it has a subsequence $\left\{M_{k}\right\}$ which converges locally uniformly on some neighborhood $V$ of 0 to some meromorphic map $g$. Since $g(0)=0, g^{\prime}(0)$ is finite. On the other hand, $g^{\prime}(0)=\lim _{k \rightarrow \infty}\left(M_{k}\right)^{\prime}(0)$. But for each positive integer $k,\left(M_{k}\right)^{\prime}(0) \geq C^{k}$. This implies $g^{\prime}(0)=\infty$ which is a contradiction. Thus 0 belongs to the Julia set of $\left\{M_{n}\right\}$, or $\infty$ is in the Julia set $\mathbf{J}$ of $\left\{N_{n}\right\}$.

Lemma 1 For every attracting fixed point $\zeta$ of $\left\{N_{P_{0}, h_{0}}^{n}\right\}$ there exist some $r>$ 0 and a neighborhood $U_{\varepsilon_{1}}\left(P_{0}\right) \times\left(\max \left\{0, h_{0}-\varepsilon_{1}\right\}, \min \left\{1, h_{0}+\varepsilon_{1}\right\}\right]$ of $\left(P_{0}, h_{0}\right)$ 
in $\mathcal{R}_{d} \times(0,1]$ such that for every sequence $\left\{\left(P_{n}, h_{n}\right)\right\}$ in this neighborhood, $D_{r}(\zeta)$ is contained in the Fatou set $\mathbf{F}$ of the family $\left\{N_{n}\right\}$, and the Julia set $\mathbf{J}$ of this family is nowhere dense.

Remark 1 In the proof we do not use the (sub-)hyperbolicity. The lemma is valid without condition (2) on $N_{P_{0}, h_{0}}$ (sec. 3.1).

\section{Proof.}

Let $\zeta$ be an attracting fixed point of $\left\{N_{P_{0}, h_{0}}^{n}\right\}$ with the immediate basin $A(\zeta)$ (such a point exists because $P_{0}$ satisfies condition 1 , sec. 3.1 , and $\left.h_{0} \neq 0\right)$. From the definition, $\left\{N_{P_{0}, h_{0}}^{n}\right\}$ converges uniformly to $\zeta$ in $A(\zeta)$, i.e. there are $q \in(0,1)$ and $r>0$ such that

$$
\forall z \in D_{r}(\zeta) \subset \subset A(\zeta):\left|\left(N_{P_{0}, h_{0}}\right)^{\prime}(z)\right| \leq q
$$

Therefore $N_{P_{0}, h_{0}}\left(D_{r}(\zeta)\right) \subset D_{r q}(\zeta) \subset \subset D_{r}(\zeta)$.

Since $N_{P, h}$ is continuous with respect to $\mathrm{P}$ and $\mathrm{h}$, there is some $\varepsilon_{1} \in\left(0, \varepsilon_{0}\right]$ such that for every polynomial $\mathrm{P}$ in $U_{\varepsilon_{1}}\left(P_{0}\right)$ and any $\mathrm{h}$ in $D_{\varepsilon_{1}}\left(h_{0}\right)$ we have

$$
N_{P, h}\left(\overline{D_{r}(\zeta)}\right) \subset D_{r}(\zeta)
$$

But $D_{r}(\zeta)$ is a nonempty open subset of $\overline{\mathbf{C}}$ with a complement which has more than three points, so from the Theorem of Montel (Theorem 5, sec. 2.1.2) and Theorem 28 (sec. 2.3) the proof is complete.

Remark 2 Since the number of (super-)attracting fixed points of $\left\{N_{P_{0}, h 0}^{n}\right\}$ is finite, we can choose $\varepsilon_{1}>0$ so that the two neighborhoods $U_{\varepsilon_{1}}\left(P_{0}\right) \subset \mathcal{R}_{d}$ and $\left(\max \left\{0, h_{0}-\varepsilon_{1}\right\}, \min \left\{1, h_{0}+\varepsilon_{1}\right\}\right] \subset(0,1]$ satisfy the previous lemma for every attracting fixed point of $\left\{N_{P_{0}, h 0}^{n}\right\}$. 
Remark 3 From the last theorem of section 2.3 we know that every repelling fixed point of the family $\left\{N_{n}\right\}$ is in the Julia set $\mathbf{J}$.

Theorem 2 If the family $\left\{N_{n}\right\}$ is chosen as in the previous lemma, then its Julia set $\mathbf{J}$ is a perfect set.

\section{Proof.}

If $z_{0} \in \mathbf{J}$ is an isolated point of $\mathbf{J}$, then there is an open neighborhood $V$ of $z_{0}$ such that $V \cap \mathbf{J}=\left\{z_{0}\right\}$ and $V \backslash\left\{z_{0}\right\} \subset \mathbf{F}$. From our assumptions on $P_{0}$, there are two different points $\zeta_{1}, \zeta_{2}$ in the set $P_{0}^{-1}(0)$. Since $\zeta_{1}, \zeta_{2}$ are attracting fixed points for $\left\{N_{P_{0}, h_{0}}^{n}\right\}$, we can find numbers $r_{1}, r_{2}>0$ so that:

- $D_{r_{1}}\left(\zeta_{1}\right) \cap D_{r_{2}}\left(\zeta_{2}\right)=\emptyset$,

- for $j=1,2$ and every positive integer $n$ :

$$
N_{n}\left(D_{r_{j}}\left(\zeta_{j}\right)\right) \subset \subset D_{r_{j}}\left(\zeta_{j}\right) \subset \subset A^{*}\left(\zeta_{j}\right)
$$

where $A^{*}\left(\zeta_{j}\right)$ is the standard immediate basin of the attracting fixed point $\zeta_{j}$ under iterations of the rational map $N_{P_{0}, h_{0}}$.

There are also positive integers $n_{1}, n_{2}$ sufficiently large such that

$$
N_{n_{j}}(V) \cap D_{r_{j}}\left(\zeta_{j}\right) \neq \emptyset,
$$

otherwise the family $\left\{N_{n}\right\}$ omits at least three different values (for example in $\left.D_{r_{1}}\left(\zeta_{1}\right)\right)$ on the domain $V$ which enables us to deduce from Montel's Theorem that $V$ is a subset of the Fatou set $\mathbf{F}$ of the family $\left\{N_{n}\right\}_{n \in \mathbf{N}}$. This contradicts $V \cap \mathbf{J}=\left\{z_{0}\right\}$.

If $W_{j}$ are defined as $W_{j}=N_{n_{j}}^{-1}\left(N_{n_{j}}(V) \cap D_{r_{j}}\left(\zeta_{j}\right)\right) \cap V$ for $j=1,2$, then each $W_{j}$ is an open subset of $V$. Therefore by taking $n_{0}=\max \left(n_{1}, n_{2}\right)$, we have 


$$
N_{n}\left(W_{j}\right) \subset D_{r_{j}}\left(\zeta_{j}\right), \quad \forall n \geq n_{0}
$$

Hence $N_{n_{0}}\left(V \backslash\left\{z_{0}\right\}\right) \cap D_{r_{j}}\left(\zeta_{j}\right) \neq \emptyset, j=1,2$ (Figure 1, below). On the other hand, we know that the derivative sequence $\left\{N_{n}^{\prime}\right\}$ is a normal family on $V \backslash\left\{z_{0}\right\}$ [Ahlfors, p. 225]. This sequence converges to 0 on the subdomains $W_{j}, j=1,2$. Therefore by Vitali's theorem the sequence $\left\{N_{n}^{\prime}\right\}$ converges locally uniformly to 0 on $V \backslash\left\{z_{0}\right\}$. Consequently, each limit function of $\left\{\left.N_{n}\right|_{W_{j}}\right\}$ is a constant contained in $D_{r_{j}}\left(\zeta_{j}\right)$.

But $V \backslash\left\{z_{0}\right\} \subset \mathbf{F}$, therefore there is a subsequence $\left\{N_{n_{k}}\right\}$ converging to a meromorphic limit function locally uniformly on $V \backslash\left\{z_{0}\right\}$ so that this limit function is constant on each $W_{j}$. We obtain $\left.N_{n_{k}}\right|_{W_{j}} \rightarrow \xi_{j} \in D_{r_{j}}\left(\zeta_{j}\right)$. By the identity theorem, $\xi_{1}=\xi_{2}$ which contradicts the fact $D_{r_{1}}\left(\zeta_{1}\right) \cap D_{r_{2}}\left(\zeta_{2}\right)=\emptyset$. Therefore $z_{0}$ is not an isolated point of $\mathbf{J}$.

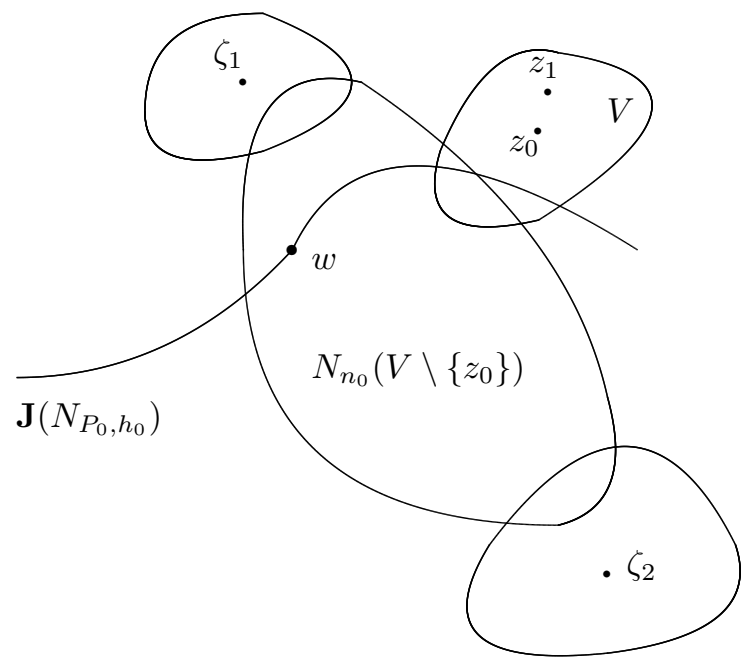

Figure 1 
Remark 4 The last theorem is also valid in general, i. e. without the (sub)hyperbolicity assumption on $N_{P_{0}, h_{0}}$.

Theorem 3 There exists some positive number $\eta>0$ so that if $\left(P_{n}, h_{n}\right) \in$ $U_{\eta}\left(P_{0}\right) \times\left(\max \left\{0, h_{0}-\eta\right\}, \min \left\{1, h_{0}+\eta\right\}\right]$ for positive integers $n$, then $\overline{O^{-}(\infty)}=$ J. The set $\overline{O^{-}(\infty)}$ is the closure of the set of the backward images of $\infty$ under the family $\left\{N_{n}\right\}, N_{n}=N_{P_{n}, h_{n}} \circ \cdots \circ N_{P_{1}, h_{1}}$.

\section{Proof.}

As we know $\infty \in \mathbf{J}\left(N_{P_{0}, h_{0}}\right)$, and $\overline{O_{N_{P_{0}, h_{0}}}^{-}(\infty)}=\mathbf{J}\left(N_{P_{0}, h_{0}}\right)$ (Theorem 11, sec. 2.2). Thus there is some positive integer $m_{0}>0$ such that $\sharp\left(N_{P_{0}, h_{0}}^{-m_{0}}(\infty)\right) \geq$ 3. We take three different complex numbers $a_{0}, b_{0}, c_{0}$ in $\left\{N_{P_{0}, h_{0}}^{-m_{0}}(\infty)\right\}$ and choose open neighborhoods $U, V, W$ at $a_{0}, b_{0}$ and $c_{0}$ respectively, so that $\bar{U}, \bar{V}, \bar{W}$ are mutually disjoint (Figure 2). From the continuity, there is some $\eta>0$ such that for every finite sequence $\left(\left(P_{n+1}, h_{n+1}\right), \cdots,\left(P_{n+m_{0}}, h_{n+m_{0}}\right)\right)$ in $U_{\eta}\left(P_{0}\right) \times\left(\max \left\{0, h_{0}-\eta\right\}, \min \left\{1, h_{0}+\eta\right\}\right]$ there are three points $a_{n}, b_{n}, c_{n}$ in $\left(N_{P_{n+m_{0}}, h_{n+m_{0}}} \circ \cdots \circ N_{P_{n+1}, h_{n+1}}\right)^{-1}(\infty)$ with $a_{n} \in U, b_{n} \in V$ and $c_{n} \in W$, where $n \in\{0,1,2, \cdots\}$.

Let $\mathrm{D}$ be a domain with $D \cap \mathbf{J} \neq \emptyset$. If for all but finitely many positive integers $n, a_{n}, b_{n}$ and $c_{n}$ are not in $N_{n}(D)$, then according to the weaker theorem of Montel (Theorem 6, sec. 2.1.2) $D \subset \mathbf{F}$, which is a contradiction. Thus there are some $z_{0} \in D$ and some positive integer $n_{0}$ such that for example $N_{n_{0}}\left(z_{0}\right)=a_{n_{0}}$. This means that $N_{n_{0}+m_{0}}\left(z_{0}\right)=\infty$ and therefore, $z_{0} \in O^{-}(\infty)$ 


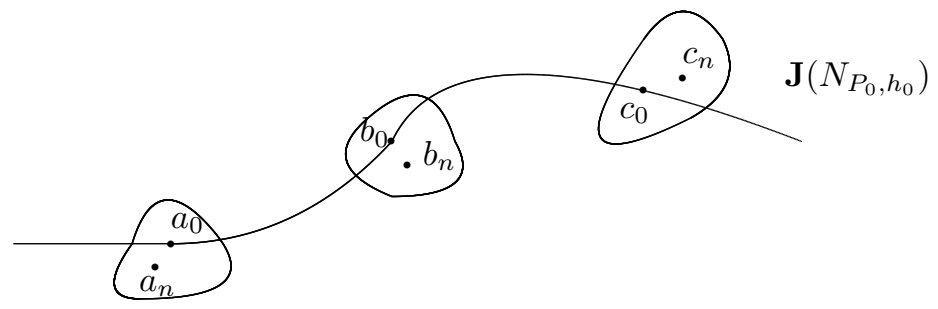

Figure 2

To have a neighborhood of $\left(P_{0}, h_{0}\right)$ where all of the theorems proven so far in this chapter are valid, we define $\varepsilon_{2}$ to be the positive number $\min \left(\eta, \varepsilon_{1}\right)$ ( $\eta$ comes from the last theorem). Our suitable neighborhood will be then the set $U_{\varepsilon_{2}}\left(P_{0}\right) \times\left(\max \left\{0, h_{0}-\varepsilon_{2}\right\}, \min \left\{1, h_{0}+\varepsilon_{2}\right\}\right]$.

\subsubsection{The Stable Domains}

First we recall that the set $\mathcal{G}(D)$ denotes the set of limit functions of all possible convergent subsequences of the family $\left\{N_{n}\right\}$ on a domain $D$. If all of the elements of $\mathcal{G}(D)$ are constant functions, then $D$ is called a contracting domain. 
Lemma 2 Let $\left\{\left(P_{n}, h_{n}\right)\right\}_{n \in \mathbf{N}}$ be in $U_{\varepsilon_{2}}\left(P_{0}\right) \times\left(\max \left\{0, h_{0}-\varepsilon_{2}\right\}, \min \left\{1, h_{0}+\right.\right.$ $\left.\varepsilon_{2}\right\}$ ]. If $\zeta$ is a repelling fixed point of the family $\left\{N_{n}\right\}$, then $\zeta \notin \mathcal{G}(D)$ for every stable domain $D$.

\section{Proof.}

Let $\mathrm{D}$ denote the given stable domain and $\zeta \in \overline{\mathbf{C}}$ be a repelling fixed point of the family such that $\zeta \in \mathcal{G}(D)$. There is a subsequence $\left\{N_{k}\right\}$ of $\left\{N_{n}\right\}$ which converges to $\zeta$ on D. If there is some $k_{0} \in \mathbf{N}$ such that $N_{k_{0}} \equiv \zeta$ on D, then $\operatorname{deg} N_{k_{0}} \equiv 0$ which contradicts the fact $\operatorname{deg} N_{k_{0}} \equiv d^{k_{0}}$.

Therefore the subsequence $\left\{N_{k}\right\}$ must be infinite. For an arbitrary $z_{0} \in D$ we define $z_{k}=N_{k}\left(z_{0}\right)$. Thus $\left(z_{k}\right)$ converges to $\zeta$. But $\left(z_{k}\right)$ is an infinite sequence, otherwise for some $\mathrm{m}, z_{m}=N_{m}\left(z_{0}\right)=\zeta$, which means $z_{0} \in N_{m}^{-1}(\zeta)=$ $N_{m}^{-1}\left(N_{m}(\zeta)\right)$ since $\zeta$ is a fixed point of the family. This would imply $z_{0} \in \mathbf{J}$ (Theorem 8, sec. 2.3), which is a contradiction to the assumption $z_{0} \in D$.

Thus $z_{k} \neq \zeta$ for every $\mathrm{k}$, and for infinitely many $\mathrm{k}$ :

$$
\left|z_{k+1}-\zeta\right|<\left|z_{k}-\zeta\right|
$$

However, we can find a number $c_{k+1}>1$ so that $\left|\left(N_{P_{k+1}, h_{k+1}}\right)^{\prime}(\zeta)\right| \geq$ $c_{k+1}>1$. Hence in some neighborhood $\mathrm{V}$ of $\zeta$ :

$$
\left|N_{P_{k+1}, h_{k+1}}(z)-\zeta\right|=\left|N_{P_{k+1}, h_{k+1}}(z)-N_{P_{k+1}, h_{k+1}}(\zeta)\right| \geq c_{k+1}|z-\zeta|
$$

for every $z \in V$. By taking $z=z_{k}$ for suitably large $\mathrm{k}$, we come to a contradiction. Thus $\zeta \notin \mathcal{G}(D)$.

So the point $\infty$ for example can not be an element of $\mathcal{G}(D)$ for every stable domain $D$ of the Fatou set $\mathbf{F}$.

Indeed, we can prove a stronger 
Lemma 3 Let $\left\{\left(P_{n}, h_{n}\right)\right\}_{n \in \mathbf{N}}$ be as in the previous lemma. If $V$ is a contracting domain, then each element of $\mathcal{G}(D)$ belongs to the Fatou set $\mathbf{F}$ of the family $\left\{N_{n}\right\}$.

\section{Proof.}

Let $V$ be a contracting domain, and $\left\{N_{n_{k}}\right\}_{k \in \mathbf{N}}$ be a subsequence of $\left\{N_{n}\right\}$ which converges on $V$ to some element $\zeta$ in $\mathcal{G}(V)$. First we claim that every $N_{n_{k}}(V)$ is a subset of $\mathbf{F}$ : otherwise there is some $v_{0} \in N_{n_{k}}(V)$ such that $v_{0} \in \mathbf{J}$. As $N_{n_{k}}(V)$ is an open set, and $O^{-}(\infty)$ is dense in the Julia set $\mathbf{J}$, there are some positive integer $m_{0}$ and some complex number $w_{0} \in N_{n_{k}}(V)$ such that $w_{0} \in N_{m_{0}}^{-1}(\infty)$. Since the point $\infty$ is a fixed point of the family $\left\{N_{n}\right\}$, this means that for some positive integer $k_{0}$ :

$$
N_{n_{j}}\left(w_{0}\right)=\infty, \forall j \geq k_{0}
$$

At least one preimage of $N_{n_{k}}^{-1}\left(w_{0}\right)$ is in $V$. Hence we deduce that $\zeta=\infty$ which is a contradiction according to the previous lemma. Therefore for every positive integer $k$ we have $N_{n_{k}}(V) \subset \mathbf{F}$, and there is some stable domain $W_{k}$ which contains $N_{n_{k}}(V)$.

Next we claim that $N_{n_{k}}(V)$ is the whole stable domain $W_{k}$; otherwise there is some positive integer $k_{0}$ such that $\partial N_{n_{k_{0}}}(V) \cap W_{k_{0}} \neq \emptyset$. Let $v_{0}$ be in $\partial N_{n_{k_{0}}}(V) \cap W_{k_{0}}$. As $V$ is a connected component of $\left(N_{n_{k_{0}}}\right)^{-1}\left(N_{n_{k_{0}}}(V)\right)$ we see that $N_{n_{k_{0}}}(\partial V)=\partial N_{n_{k_{0}}}(V)$. Therefore there is some $z_{0} \in \partial V \subset \mathbf{J}$ such that $v_{0}=N_{n_{k_{0}}}\left(z_{0}\right)$. On the other hand, there is some open neighborhood $U_{0}$ of $v_{0}$ in $W_{k_{0}}$. Hence $U_{0} \cap N_{n_{k_{0}}}(\partial V) \neq \emptyset$. As $\overline{O^{-}(\infty)}=\mathbf{J}$, we can find some positive integer $m_{0}$ so that $N_{m_{0}}^{-1}(\infty) \in N_{n_{k_{0}}}^{-1}\left(U_{0} \cap N_{n_{k_{0}}}(\partial V)\right)$, i. e. $N_{n_{k_{0}}}\left(N_{m_{0}}^{-1}(\infty)\right) \in N_{n_{k_{0}}}(\partial V) \cap W_{k_{0}}$. For every positive integer $k$ we have $N_{n_{k}}(V) \subset W_{k}$, according to the definition of $W_{k}$. Therefore for every positive integer $m$ : 


$$
N_{n_{k_{0}+m}}\left(N_{m_{0}}^{-1}(\infty)\right) \in \bar{W}_{k_{0}+m} \cap N_{n_{k_{0}+m}}(\partial V)
$$

This yields either $\infty \in W_{k_{0}+m}$ which is a contradiction $\left(W_{k_{0}+m}\right.$ is a stable domain), or $\infty \in \partial\left(W_{k_{0}+m}\right) \cap \partial N_{n_{k_{0}+m}}(V)$. But $\overline{N_{n_{k_{0}+m}}(V)} \longrightarrow \zeta$ as $m \longrightarrow \infty$ which gives $\zeta=\infty$, again a contradiction according to the previous lemma.

Therefore $N_{n_{k}}(V)=W_{k}$ for every positive integer $k$. We deduce there is some positive integer $k_{0}$ so that $\zeta \in N_{n_{k_{0}}}(V)$, otherwise the previous paragraph gives the contradiction $\zeta=\infty$ again!

From the above proof we have an important result which we formulate in the following

Theorem 4 Let $\left\{\left(P_{n}, h_{n}\right)\right\}_{n \in \mathbf{N}}$ be as in Lemma 2, and $V$ be a contracting domain of the Fatou set $\mathbf{F}$ of the related family $\left\{N_{n}\right\}_{n \in \mathbf{N}}$. If $\left\{N_{n_{k}}\right\}$ is a subsequence of $\left\{N_{n}\right\}$ convergent to an element $\zeta \in \mathbf{C}$ of the limit set $\mathcal{G}(V)$, then each set $N_{n_{k}}(V)$ is a stable domain of $\mathbf{F}$. Further, there is some positive integer $k_{0}$ such that $\zeta \in N_{n_{k_{0}}}(V), N_{n_{k_{0}}}(V)$ remains invariant under $N_{P_{k}, h_{k}}$ 's for $k>k_{0}$ and has therefore $\infty$ on its boundary $\partial N_{n_{k_{0}}}(V)$.

Notation 1 In what follows, we denote the real interval ( $\max \left\{0, h_{0}-\varepsilon_{2}\right\}, \min \left\{1, h_{0}+\right.$ $\left.\left.\varepsilon_{2}\right\}\right]$ briefly by $D_{\varepsilon_{2}}\left(h_{0}\right)$.

Definition 1 A stable domain $V$ of the Fatou set $\mathbf{F}$ is called a "wandering" domain if $N_{m}(V) \cap N_{n}(V)=\emptyset$ for every distinct positive integers $m$ and $n$. For $n=0$ we assume $N_{0}(V)=V$. We denote by $\mathcal{W}$ the set of the points $z \in \overline{\mathbf{C}}$ with the property that each $z \in \mathcal{W}$ has an open "wandering" neighborhood $U_{z}$, i. e. with the property $N_{m}\left(U_{z}\right) \cap N_{n}\left(U_{z}\right)=\emptyset$, for all positive integers $m, n$. The complement of $\mathcal{W}$ is denoted by $\mathcal{A}$. 
The set $\mathcal{W}$ is an open set according to the definition and therefore $\mathcal{A}$ a closed one.

The Julia set $\mathbf{J}$ is a subset of $\mathcal{A}$ : if $z \in \mathbf{J}$, then for every open neighborhood $U$ of $z$ there exists a positive integer $m$ with the property $z_{m}=$ $\left(N_{m}\right)^{-1}(\infty) \in U$. Let $U_{1}, U_{2}$ be two such neighborhoods with $U_{2} \subset \subset U_{1}$. We can find two positive integers $m_{1} \neq m_{2}$ with $z_{2}=\left(N_{m_{2}}\right)^{-1}(\infty) \in U_{2}$ and $z_{1}=\left(N_{m_{1}}\right)^{-1}(\infty) \in U_{1}$. This means $\infty \in N_{m_{1}}\left(U_{1}\right) \cap N_{m_{2}}\left(U_{1}\right)$. As $U_{1}$ was arbitrary, $\mathbf{J}$ can not be a subset of $\mathcal{W}$.

Lemma 4 Let $W \subset \mathcal{W}$ be a domain. Then for every compact subset $K$ of $W, \operatorname{diam}\left[N_{n}(K)\right] \longrightarrow 0$ as $n \longrightarrow \infty$ ([Beardon, $p$. 17r]]).

\section{Proof.}

Suppose that this is false. Then there is a compact subset $K$ of $W$, a positive number $\alpha$, and some increasing sequence $n_{j}$ of positive integers such that for $j=1,2, \cdots$,

$$
(*) \quad \operatorname{diam}\left[N_{n}(K)\right] \geq \alpha .
$$

Since $\mathbf{J} \subset \mathcal{A}$, the family $\left\{N_{n}\right\}$ is normal in $W$. Thus there is a subsequence of $\left\{N_{n_{j}}\right\}$ which converges locally uniformly on $W$ to some meromorphic function $g$. We relabel this subsequence and assume that $\left\{N_{n_{j}}\right\}$ itself has this property. If $g$ is constant with value $c$ on $W$, then $N_{n_{j}}$ converges uniformly to $c$ on $K$, and therefore for large $j, N_{n_{j}}(K)$ lies in an $\frac{\alpha}{3}$-neighborhood of $c$. This contradicts $(*)$. We conclude that $g$ is non-constant on $W$.

Therefore we can find a point $\zeta \in W$ where $g^{\prime}(\zeta) \neq 0$ and drew a small circle $C$ with center $\zeta$ which with its interior $D$ lies in $W$, and which is such that $g(z) \neq g(\zeta)$ when $z$ is on the circle $C$. As $\inf _{w \in C}|g(w)-g(\zeta)|>0$, there is some positive integer $j_{0}$ such that for $j \geq j_{0}$,

$$
\left|N_{n_{j}}(z)-g(z)\right|<\inf _{w \in C}|g(w)-g(\zeta)|<|g(z)-g(\zeta)|
$$


on $C$. So by Rouché's Theorem, $N_{n_{j}}(D)$ contains the point $g(\zeta)$ for $j \geq j_{0}$ which contradicts the wandering character of $W$. This completes the proof.

From the previous lemma (Lemma 4) and Theorem 31, sec. 2.3., we deduce that each wandering stable domain $V$ is actually a contracting (stable) domain. But this contradicts the wandering nature of $V$, according to the proof of Lemma 3. In fact, for each contracting domain $V$ there are sufficiently large positive integers $m$ and $n$ such that $N_{m}(V)=N_{n}(V)$. Therefore there is no wandering stable domains in the Fatou set $\mathbf{F}$ ! 


\subsection{Convergent Sequences Near $\left(\mathrm{P}_{0}, \mathrm{~h}_{0}\right)$}

\subsubsection{Introduction}

Let $\left\{\left(P_{n}, h_{n}\right)\right\}_{n \in \mathbf{N}}$ be in $U_{\varepsilon_{2}}\left(P_{0}\right) \times D_{\varepsilon_{2}}\left(h_{0}\right)$ as before, with the extra property

$$
\mathbf{P}_{\mathbf{n}} \longrightarrow \mathbf{P}_{0} \text { and } \mathbf{h}_{\mathbf{n}} \longrightarrow \mathbf{h}_{0} \text { as } \mathbf{n} \longrightarrow \infty \text {. }
$$

We assume again that $N_{P_{0}, h_{0}}$ is a subhyperbolic rational function.

As we know so far, the Julia set $\mathbf{J}\left(N_{P_{0}, h_{0}}\right)$ of the family $\left\{N_{P_{0}, h_{0}}^{n}\right\}$ is connected and locally connected ([Milnor] $)$. Further, $\mu\left(\mathbf{J}\left(N_{P_{0}, h_{0}}\right)\right)=0$, where $\mu$ is the two-dimentional Lebesgue measure produced by the spherical metric on $\overline{\mathbf{C}}$ (Lyubich 1983). Its Fatou set $\mathbf{F}\left(N_{P_{0}, h_{0}}\right)$ consists of simply-connected contracting (stable) domains ([Milnor, Beardon]). In the following sections we try to find out if there is some $\varepsilon>0$ such that for every convergent sequence $\left\{\left(P_{n}, h_{n}\right)\right\}_{n \in \mathbf{N}}$ in $U_{\varepsilon}\left(P_{0}\right) \times D_{\varepsilon}\left(h_{0}\right)$, the Julia and Fatou sets of the family $\left\{N_{n}=N_{P_{n}, h_{n}} \circ \cdots \circ N_{P_{1}, h_{1}}\right\}$ have similar behaviours as of $\mathbf{J}\left(N_{P_{0}, h_{0}}\right)$ and $\mathbf{F}\left(N_{P_{0}, h_{0}}\right)$, respectively. 


\subsubsection{The Structure of the Fatou Set}

Theorem 5 Let $\left\{N_{n}\right\}_{n \in \mathbf{N}}, N_{n}=N_{P_{n}, h_{n}} \circ \cdots \circ N_{P_{1}, h_{1}}$, be a family in $U_{\varepsilon_{2}}\left(P_{0}\right) \times$ $D_{\varepsilon_{2}}\left(h_{0}\right)$ as mentioned in sec. 3.2.2, such that $P_{n} \longrightarrow P_{0}$ and $h_{n} \longrightarrow h_{0}$ as $n \longrightarrow \infty$. If there is a subsequence $\left\{N_{n_{k}}\right\}_{k \in \mathbf{N}}$ which converges locally uniformly on a stable domain $W \in \mathbf{F}$ to a constant map $\zeta \in \mathbf{C}$, then $\zeta$ is a (super-)attracting periodic point of $\left\{N_{P_{0}, h_{0}}^{n}\right\}$.

\section{Proof.}

First, $\zeta$ is not in $\mathbf{J}\left(N_{P_{0}, h_{0}}\right)$, since otherwise $N_{P_{0}, h_{0}}$ (which is subhyperbolic) expands in a neighborhood of $\zeta$ except perhaps at $\zeta$, and therefore for some suitable positive integer $k_{0},\left\{N_{P_{n_{k}}, h_{n_{k}}}\right\}_{k \geq k_{0}}$ expands in a neighborhood of $\zeta$, too. This contradicts the assumptions of the theorem. Thus there is some stable domain $\mathrm{V}$ of $\mathbf{F}\left(N_{P_{0}, h_{0}}\right)$ which contains $\zeta$. So we can find some $r>0$ so that $D_{r}(\zeta) \subset V$.

We know that every stable domain of $\mathbf{F}\left(N_{P_{0}, h_{0}}\right)$ is a basin of attraction for some (super-)attracting periodic point of $\left\{N_{P_{0}, h_{0}}^{n}\right\}$. If $\zeta$ is not a (super)attracting periodic point of $\left\{N_{P_{0}, h_{0}}^{n}\right\}$, then there exists some other point $\eta \neq \zeta$ in $V$ such that for a subsequence of the family which we denote also by $\left\{N_{P_{0}, h_{0}}^{m}\right\}_{m \in \mathbf{N}}$ we have $\lim _{m \rightarrow \infty} N_{P_{0}, h_{0}}^{m}=\eta$ locally uniformly on $V$. Therefore for each neighborhood $D_{\varepsilon}(\eta)$ of $\eta$ there is a neighborhood $D_{\delta}(\zeta)$, and some $M_{0} \in \mathbf{N}$ such that

$$
(*) \quad N_{P_{0}, h_{0}}^{m}\left(D_{\delta}(\zeta)\right) \subset D_{\varepsilon}(\eta), \quad \forall m \geq M_{0} .
$$

Now let $K$ be a nonempty compact subset of $W$. There is some subsequence $\left\{N_{n_{k}}\right\}$ of $\left\{N_{n}\right\}$ which is convergent on $K$ to $\zeta$. Hence there is some 
positive integer $M_{1}$ such that for every $z \in K$ and every $k \geq M_{1}$ :

$$
\left|N_{n_{k}}(z)-\zeta\right|<\frac{\delta}{2}
$$

We can choose $\delta, \varepsilon>0$ so that $D_{\delta}(\zeta) \cap D_{\varepsilon}(\eta)=\emptyset$. On the other hand, for every positive integer $l$ there is some positive integer $M_{l}$ with

$$
\left|N_{P_{n_{k}+l}, h_{n_{k}+l}} \circ \cdots \circ N_{P_{n_{k}+1}, h_{n_{k}+1}}(z)-N_{P_{0}, h_{0}}^{l}(z)\right|<\frac{\delta}{4}
$$

for every $z$ in $\overline{\mathbf{C}}$, and every $k \geq M_{l}$.

Let $M=\max \left(M_{l}, M_{1}\right)$ and $z_{l}=N_{P_{n_{M}+l}, h_{n_{M}+l}} \circ \cdots \circ N_{P_{n_{M}+1}, h_{n_{M}+1}}\left(z_{1}\right)$ for some $z_{1}=N_{n_{M}}\left(z_{0}\right)$, where $z_{0} \in K$. Then $z_{l} \in D_{\frac{\delta}{2}}(\zeta) \subset D_{\delta}(\zeta)$ and

$$
\begin{gathered}
\left|N_{P_{0}, h_{0}}^{l}\left(z_{l}\right)-\zeta\right| \leq \\
\left|N_{P_{0}, h_{0}}^{l}\left(z_{l}\right)-N_{P_{n_{M}+l}, h_{n_{M}+l}} \circ \cdots \circ N_{P_{n_{M}+1}, h_{n_{M}+1}}\left(z_{1}\right)\right|+ \\
\left|N_{P_{n_{M}+l}, h_{n_{M}+l}} \circ \cdots \circ N_{P_{n_{M}+1}, h_{n_{M}+1}}\left(N_{n_{M}}\left(z_{0}\right)\right)-\zeta\right| \leq \\
\frac{\delta}{4}+\left|N_{n_{M}+l}\left(z_{0}\right)-\zeta\right| \leq \quad \frac{\delta}{4}+\frac{\delta}{2}<\delta .
\end{gathered}
$$

So we have shown that for every positive integer $l$ there is some $z_{l} \in D_{\delta}(\zeta)$ such that $N_{P_{0}, h_{0}}^{l}\left(z_{l}\right)$ remains in $D_{\delta}(\zeta)$. Since $D_{\delta}(\zeta)$ and $D_{\varepsilon}(\eta)$ are disjoint, this contradicts $\left(^{*}\right)$. Thus $\zeta$ is a periodic point of $\left\{N_{P_{0}, h_{0}}^{n}\right\}$.

Corollary 1 If $V$ is a contracting domain of $\left\{N_{n}\right\}$, then $\mathcal{G}(V)=O^{+}(\zeta)$ for some periodic point $\zeta \in \mathbf{C}$.

\section{Proof.}

Let $K$ be a compact subset of $V$. If $\zeta$ and $\eta$ are two different complex values in $\mathcal{G}(V)$, then we can find two subsequences $\left\{N_{i}\right\}$ and $\left\{N_{j}\right\}$ of $\left\{N_{n}\right\}$ with 
$N_{i} \longrightarrow \zeta$ and $N_{j} \longrightarrow \eta$ as $i \longrightarrow \infty$ and $j \longrightarrow \infty$, respectively. Therefore there are disjoint neighborhoods $U_{\zeta}$ at $\zeta$ and $U_{\eta}$ at $\eta$ such that for some positive integers $i_{0}$ and $j_{0}, N_{i}(K) \subset U_{\zeta}$ and $N_{j}(K) \subset U_{\eta}$, for some $i \geq i_{0}$ and some $j \geq j_{0}$ respectively. Since $\zeta$ and $\eta$ are two different periodic points of $\left\{N_{P_{0}, h_{0}}\right\}$, we can find two disjoint neighborhoods $V_{\zeta} \subset U_{\zeta}$ and $V_{\eta} \subset U_{\eta}$ at $\zeta$ and $\eta$ respectively, and some positive integer $n_{0}$ so that $N_{P_{n}, h_{n}}\left(V_{\zeta}\right) \subset V_{\zeta}$ and $N_{P_{n}, h_{n}}\left(V_{\eta}\right) \subset V_{\eta}$ for every $n>n_{0}$. Therefore $N_{n}(K) \subset V_{\zeta} \cap V_{\eta}, \forall n>$ $\max \left(n_{0}, i_{0}, j_{0}\right)$, which contradicts our assumption that $V_{\zeta} \cap V_{\eta}=\emptyset$.

Notation 2 Let $\zeta$ be a complex number. The set $B(\zeta)$ denotes the contracting domain for which $\{\zeta\} \in \mathcal{G}(B(\zeta))$. The number $\zeta$ is called a contracting value of the family $\left\{N_{n}\right\}$.

Recall 1 The number of attracting periodic points for the family $\left\{N_{P_{0}, h_{0}}^{n}\right\}$ is a finite number $s>0$, according to Riemann-Hurewitz Theorem. Therefore there are $s$ distinct contracting values $\zeta_{i}, 1 \leq i \leq s$, for the family $\left\{N_{n}\right\}$, where $1 \leq s<\infty$. The positive integer $d$ is the degree of $N_{P_{0}, h_{0}}$. If we denote the set

$\left\{z \in \mathbf{C} \mid \lim _{k \rightarrow \infty} N_{P_{0}, h_{0}}^{n_{k}}(z)=\alpha_{j}\right.$, where $\alpha_{j}$ is some attracting periodic point equal to $\zeta_{i}, 1 \leq j \leq t$ and $\left.s \leq t<\infty\right\}$

by $A\left(\zeta_{i}\right)$, then $\mathbf{F}\left(N_{P_{0}, h_{0}}\right)=\bigcup_{i=1}^{t} A\left(\zeta_{i}\right)$.

Definition 2 For every $\xi>0$, the $\xi$-neighborhood of a set $A$ in $\overline{\mathbf{C}}$ is

$$
U_{\xi}(A)=\{z \in \overline{\mathbf{C}} \mid \chi(z, A) \leq \xi\}
$$

where $\chi$ is the spherical distance. 
Lemma 5 For every $\eta>0$ there is some $\epsilon \in\left(0, \varepsilon_{2}\right]$ such that for every $\left\{\left(P_{n}, h_{n}\right)\right\}_{n \in \mathbf{N}}$ in $U_{\epsilon}\left(P_{0}\right) \times D_{\epsilon}\left(h_{0}\right)$,

$$
\mathbf{F} \backslash \bigcup_{i=1}^{s} B\left(\zeta_{i}\right) \subset U_{\eta}\left(\mathbf{J}\left(N_{P_{0}, h_{0}}\right)\right)
$$

where each $\zeta_{i}$ is an attracting periodic point of the family $\left\{N_{P_{0}, h_{0}}^{n}\right\}$, and $1 \leq$ $s<\infty$. The set $\mathbf{F}$ is the Fatou set defined for the family $\left\{N_{n}\right\}$, where $\left\{\left(P_{n}, h_{n}\right)\right\}$ are now in $U_{\epsilon}\left(P_{0}\right) \times D_{\epsilon}\left(h_{0}\right)$.

\section{Proof.}

Since for every $\eta>0, \overline{\mathbf{C}} \backslash U_{\eta}\left(\mathbf{J}\left(N_{P_{0}, h_{0}}\right)\right)$ is compact, there is some $r>0$ and some positive integer $m$ with

$$
N_{P_{0}, h_{0}}^{m}\left(\overline{\mathbf{C}} \backslash U_{\eta}\left(\mathbf{J}\left(N_{P_{0}, h_{0}}\right)\right)\right) \subset \bigcup_{i=1}^{s} D_{r}\left(\zeta_{i}\right), \quad \text { for some } s<\infty .
$$

From the continuity with respect to the norm on the space of rational maps we deduce that there is some $\epsilon \in\left(0, \varepsilon_{2}\right]$ such that

$$
N_{P, h}\left(\bigcup_{i=1}^{s} D_{r}\left(\zeta_{i}\right)\right) \subset \subset \bigcup_{i=1}^{s} D_{r}\left(\zeta_{i}\right),
$$

and

$$
N_{P_{m}, h_{m}} \circ \cdots \circ N_{P_{1}, h_{1}}\left(\mathbf{F}\left(N_{P_{0}, h_{0}}\right) \backslash U_{\eta}\left(\mathbf{J}\left(N_{P_{0}, h_{0}}\right)\right)\right) \subset \subset \bigcup_{i=1}^{s} D_{r}\left(\zeta_{i}\right),
$$

where $(P, h)$ and $\left(P_{n}, h_{n}\right)$ are in $U_{\epsilon}\left(P_{0}\right) \times D_{\epsilon}\left(h_{0}\right)$.

So from Theorem 8, sec. 2.2:

$$
\overline{\mathbf{C}} \backslash U_{\eta}\left(\mathbf{J}\left(N_{P_{0}, h_{0}}\right)\right) \subset \mathbf{F} .
$$

Since $\mathbf{F}\left(N_{P_{0}, h_{0}}\right) \cup \mathbf{J}\left(N_{P_{0}, h_{0}}\right)=\overline{\mathbf{C}}$, 


$$
\mathbf{F} \backslash \bigcup_{i=1}^{s} B\left(\zeta_{i}\right) \subset \mathbf{F} \backslash \bigcup_{i=1}^{s} D_{r}\left(\zeta_{i}\right) \subset U_{\eta}\left(\mathbf{J}\left(N_{P_{0}, h_{0}}\right)\right)
$$

We choose $\varepsilon_{2}$ equal to the positive number $\epsilon$ in the previous lemma.

Lemma 6 Assume $N_{P_{0}, h_{0}}$ to be subhyperbolic as before. Then for every c $>1$ there is a neighborhood $U$ of $\mathbf{J}\left(N_{P_{0}, h_{0}}\right)$ and a positive integer $n_{0}$ such that for every $n \geq n_{0}$

$$
\sup _{z \in U}\left|\left(N_{P_{n}, h_{n}} \circ \cdots \circ N_{P_{n_{0}}, h_{n_{0}}}\right)^{\prime}(z)\right| \geq c .
$$

\section{Proof.}

From the definition of subhyperbolicity there is a neighborhood $U$ of the Julia set $\mathbf{J}\left(N_{P_{0}, h_{0}}\right)$, where the rational function $N_{P_{0}, h_{0}}$ is expanding except at finitely many points in $U$. Therefore for every $s>1$ there is some positive integer $K$ such that

$$
\sup _{z \in U}\left|\left(N_{P_{0}, h_{0}}^{n}\right)^{\prime}(z)\right| \geq s, \forall n \geq K
$$

(the critical orbits in $\mathbf{J}\left(N_{P_{0}, h_{0}}\right)$ are finite).

On the other hand, $\left\{N_{P_{n}, h_{n}}\right\}$ converges locally uniformly on compact subsets to $N_{P_{0}, h_{0}}$, and $\mathbf{J}\left(N_{P_{0}, h_{0}}\right)$ is invariant under $N_{P_{0}, h_{0}}$. Thus for every $\delta>0$ there is some positive integer $L$ such that on $U$ :

$$
\left|\left(N_{P_{n+K-1}, h_{n+K-1}} \circ \cdots \circ N_{P_{n}, h_{n}}\right)^{\prime}(z)-\left(N_{P_{0}, h_{0}}^{K}\right)^{\prime}(z)\right| \leq \delta, \forall n \geq L .
$$

We can also modify $L$ so that for every $n \geq L$,

$$
\left|\left(N_{P_{n}, h_{n}}\right)^{\prime}-\left(N_{P_{0}, h_{0}}\right)^{\prime}\right|<\delta,
$$

on compact subsets.

Now we can choose $n_{0}:=\max (L, K)$. Thus for every $n \geq n_{0}$ : 


$$
\begin{aligned}
& \sup _{z \in U}\left|\left(N_{P_{n}, h_{n}} \circ \cdots \circ N_{P_{n_{0}}, h_{n_{0}}}\right)^{\prime}(z)\right|= \\
& \sup _{z \in U}\left[\left|\left(N_{P_{n}, h_{n}} \circ \cdots \circ N_{P_{n_{0}+K}, h_{n_{0}+K}}\right)^{\prime}\left(N_{P_{n_{0}+K-1}, h_{n_{0}+K-1}} \circ \cdots \circ N_{P_{n_{0}}, h_{n_{0}}}\right)(z)\right| \cdot\right. \\
& \left.\left|\left(N_{P_{n_{0}+K-1}, h_{n_{0}+K-1}} \circ \cdots \circ N_{P_{n_{0}}, h_{n_{0}}}\right)^{\prime}(z)\right|\right] \geq \\
& (s-\delta)^{n-\left(n_{0}+K\right)-1} \sup \left(\left|\left(N_{P_{0}, h_{0}}^{K}\right)^{\prime}(z)\right|-\right. \\
& \left|\left(N_{P_{n_{0}+K-1}, h_{n_{0}+K-1}} \circ \cdots \circ N_{P_{n_{0}}, h_{n_{0}}}\right)^{\prime}(z)-\left(N_{P_{0}, h_{0}}^{K}\right)^{\prime}(z)\right| \geq(s-\delta)^{2} .
\end{aligned}
$$

The number $\delta>0$ can be chosen so small that $(s-\delta)^{2}:=c$ remains greater than 1 . Hence for every $n \geq n_{0}$ :

$$
\sup _{z \in U}\left|\left(N_{P_{n}, h_{n}} \circ \cdots \circ N_{P_{n_{0}}, h_{n_{0}}}\right)^{\prime}(z)\right| \geq c>1
$$

Theorem 6 For every convergent sequence $\left\{\left(P_{n}, h_{n}\right)\right\} \subset U_{\varepsilon_{2}}\left(P_{0}\right) \times D_{\varepsilon_{2}}\left(h_{0}\right)$ there is positive integers $s$ and $k$ such that the Fatou set $\mathbf{F}$ of the family of the relaxed Newton's methods $\left\{N_{n}\right\}$ generated by $\left\{\left(P_{n}, h_{n}\right)\right\}_{n \geq k}$ consists only of contracting domains:

$$
\mathbf{F}=\bigcup_{i=1}^{s} B\left(\zeta_{i}\right)
$$

\section{Proof.}

From Lemma 6 we can find for every $c>1$ some $\xi>0$ and a positive integer $n_{0}$ so that for some $x_{1}, x_{2} \in U_{\xi}\left(\mathbf{J}\left(N_{P_{0}, h_{0}}\right)\right)$ and every $n \geq n_{0}$ :

$$
\left|N_{P_{n}, h_{n}} \circ \cdots \circ N_{P_{n_{0}}, h_{n_{0}}}\left(x_{1}\right)-N_{P_{n}, h_{n}} \circ \cdots \circ N_{P_{n_{0}}, h_{n_{0}}}\left(x_{2}\right)\right| \geq c\left|x_{1}-x_{2}\right| .
$$

From Lemma 5 there exists some positive integer $n_{1}$ with

$$
\mathbf{F}\left(\left\{N_{P_{n}, h_{n}} \circ \cdots \circ N_{P_{n_{1}}, h_{n_{1}}}\right\}_{n \geq n_{1}}\right) \backslash \bigcup B(\zeta) \subset U_{\xi}\left(\mathbf{J}\left(N_{P_{0}, h_{0}}\right)\right),
$$


where the union $\bigcup B(\zeta)$ is over all (super-)attracting periodic points $\zeta$ of the family $\left\{N_{P_{0}, h_{0}}^{n}\right\}$. Thus if $U$ is a connected open subset of $\mathbf{F}\left(\left\{N_{P_{n}, h_{n}} \circ \cdots \circ\right.\right.$ $\left.\left.N_{P_{n_{1}}, h_{n_{1}}}\right\}_{n \geq n_{1}}\right) \backslash \bigcup B(\zeta)$, then $\bar{U} \subset U_{\xi}\left(\mathbf{J}\left(N_{P_{0}, h_{0}}\right)\right)$ (from the proof of Lemma 6 ).

On the other hand, as $N_{P_{n}, h_{n}} \longrightarrow N_{P_{0}, h_{0}}$, and $\mathbf{J}\left(N_{P_{0}, h_{0}}\right)$ is invariant under $\left\{N_{P_{0}, h_{0}}^{n}\right\}$, we can find some positive integer $n_{2} \geq n_{1}$ so that for every $n \geq n_{2}$

$$
N_{P_{n}, h_{n}} \circ \cdots \circ N_{P_{n_{2}}, h_{n_{2}}}\left(U_{\xi}\left(\mathbf{J}\left(N_{P_{0}, h_{0}}\right)\right)\right) \subset U_{\xi}\left(\mathbf{J}\left(N_{P_{0}, h_{0}}\right)\right)
$$

Since $N_{P_{0}, h_{0}}$ is expanding on $\mathbf{J}\left(N_{P_{0}, h_{0}}\right)$ except at finitely many points, we can choose $x_{1}, x_{2} \in U \subset U_{\xi}\left(\mathbf{J}\left(N_{P_{0}, h_{0}}\right)\right)$ and $n_{2}$ so that for every $m \geq n_{2}$ :

$$
\left|N_{P_{m}, h_{m}}\left(x_{1}\right)-N_{P_{m}, h_{m}}\left(x_{2}\right)\right| \geq \lambda_{m}\left|x_{1}-x_{2}\right|
$$

for some $\lambda_{m}>1$. If we put $k=\max \left(n_{0}, n_{2}\right)$, then for the above $x_{1}, x_{2} \in$ $U \subset U_{\xi}\left(\mathbf{J}\left(N_{P_{0}, h_{0}}\right)\right)$ we deduce the inequality $(*)$

$$
\begin{gathered}
\mid N_{P_{n}, h_{n}} \circ \cdots \circ N_{P_{k+1}, h_{k+1}}\left(N_{P_{k}, h_{k}}\left(x_{1}\right)\right)- \\
N_{P_{n}, h_{n}} \circ \cdots \circ N_{P_{k+1}, h_{k+1}}\left(N_{P_{k}, h_{k}}\left(x_{2}\right)\right) \mid \geq \\
\lambda_{n} \cdots \lambda_{k+1}\left(c\left|x_{1}-x_{2}\right|\right) .
\end{gathered}
$$

As $x_{1}, x_{2}$ are in the connected set $U \subset \mathbf{F}\left(\left\{N_{P_{n}, h_{n}} \circ \cdots \circ N_{P_{k}, h_{k}}\right\}_{n \geq k}\right) \backslash$ $\bigcup_{i=1}^{s} B\left(\zeta_{i}\right)$ as mentioned before, the inequality $(*)$ contradicts the normality of the family $\left\{N_{P_{n}, h_{n}} \circ \cdots \circ N_{P_{s}, h_{s}}\right\}_{n \geq s}$ on $U$. Therefore

$$
\mathbf{F}=\bigcup_{i=1}^{s} B\left(\zeta_{i}\right)
$$




\subsubsection{The Measure of the Julia Set}

We intend here to show that the Julia set $\mathbf{J}$ of the convergent family $\left\{N_{n}\right\}_{n \in \mathbf{N}}$ is a set of measure zero. The measure $\mu$ is the image of the two dimentional Lebesgue measure of $\mathbf{R}^{2} \cup\{\infty\}$ under the inverse map $\pi^{-1}$ of the stereographic projection defined in sec. 2.1.1 on the Riemann's sphere $\overline{\mathbf{C}}$. The proof is almost the one given in Lyubich 1983 for the same property of the standard Julia set $\mathbf{J}\left(N_{P_{0}, h_{0}}\right)$, however with some changes in the related definitions.

In what follows, we choose the positive number $\varepsilon_{2}$ so small that Theorem 6 of the previous section holds for our family $\left\{N_{n}\right\}$ mentioned above, i. e. the Fatou set $\mathbf{F}$ of the family $\left\{N_{n}\right\}$ consists only of contracting domains. We know from sec. 3.2.3 that $\mathbf{J} \subset \mathcal{A}(\mathcal{A}$ is defined in Definition 1 , sec. 3.2.3). Indeed, each point in $\mathcal{W}$ (Definition 1, sec. 3.2.3) has a "wandering" neighborhood which goes under $\left\{N_{n}\right\}$ to some contracting value $\zeta$ of the family as $n \longrightarrow \infty$ (Lemma 4, sec. 3.2.3). Therefore each point in $\mathcal{W}$ belongs to the Fatou set, and we deduce again that $\mathcal{W} \subset \mathbf{F}$.

Notation 3 Let $C\left(N_{i}\right)$ and $C\left(N_{P_{i}, h_{i}}\right)$ denote the sets of critical points of $N_{i}$ and $N_{P_{i}, h_{i}}$, respectively. Then for $1 \leq m \leq \infty$ the set $C_{m}$ defined by

$$
C_{m}=C\left(N_{P_{1}, h_{1}}\right) \cup N_{P_{1}, h_{1}}^{-1}\left(C\left(N_{P_{2}, h_{2}}\right)\right) \cup \cdots \cup\left(N_{m-1}\right)^{-1}\left(C\left(N_{P_{m}, h_{m}}\right)\right)
$$

denotes the set of critical points of $N_{m}$. The set of critical values of $N_{m}$ will be therefore the set

$$
\begin{gathered}
Z_{m}=N_{m}\left(C\left(N_{P_{1}, h_{1}}\right)\right) \cup\left(N_{P_{m}, h_{m}} \circ \cdots \circ N_{P_{2}, h_{2}}\left(C\left(N_{P_{2}, h_{2}}\right)\right)\right) \cup \cdots \cup \\
N_{P_{m}, h_{m}}\left(C\left(N_{P_{m}, h_{m}}\right)\right) .
\end{gathered}
$$


Also, the spherical distance of the point $z$ from the set $C_{m}$ is denoted by $r_{m}(z):=\chi\left(z, C_{m}\right)$. We recall that the ball of radius $r$ at $z$ in this metric is denoted by $D_{r}(z)$.

Lemma 7 Under the above assumptions on the family $\left\{N_{n}\right\}$ we have

$$
\mathbf{J} \subset \partial \mathcal{W}
$$

\section{Proof.}

Let $z \in \mathbf{J}$ be an arbitrary point. As $\mathbf{J}$ is nowhere dense, there is some open neighborhood $U_{z}$ of $z$ with the property

$$
U_{z} \cap \mathbf{F} \neq \emptyset
$$

We can find a connected open set $V_{z}$ in $U_{z} \cap \mathbf{F}$ so that there exists a contracting domain $V \subset \mathbf{F}$ with $\overline{V_{z}} \subset V$. There is some complex number $\zeta \in \mathbf{C}$ with $\mathcal{G}(V)=\{\zeta\}$. So we can find some subsequence $\left\{N_{n_{k}}\right\}$ of $\left\{N_{n}\right\}$ which converges uniformly on $\overline{V_{z}}$ to $\zeta$ as $k$ goes to $\infty$. On the other hand, $\zeta$ is a (super-)attracting periodic point of the family $\left\{N_{P_{0}, h_{0}}^{n}\right\}$. We assume first that $\zeta$ is a (super-)attracting fixed point of $N_{P_{0}, h_{0}}$. There is some open neighborhood $D_{\zeta}$ of $\zeta$ such that $N_{P_{0}, h_{0}}\left(D_{\zeta}\right) \subset \subset D_{\zeta}$ and $\left|N_{P_{0}, h_{0}}^{\prime}(z)\right|=\lambda<1$. Let $w$ be some point in $V_{z}$ such that for every positive integer $n, N_{n}(w) \neq \zeta$, and $n_{0}$ be the smallest positive integer with $N_{n_{0}}(w) \in D_{\zeta}$. Since $N_{P_{n}, h_{n}} \longrightarrow$ $N_{P_{0}, h_{0}}$ as $n \longrightarrow \infty$, there is some positive integer $m_{0}$ such that for every $m \geq m_{0}$ :

$$
\begin{aligned}
& N_{P_{m}, h_{m}}\left(D_{\zeta}\right) \subset D_{\zeta}, \\
& \left|N_{P_{m}, h_{m}}^{\prime}(z)\right|=\lambda_{m}<1 \text { and } \\
& \lambda_{m} \longrightarrow \lambda \text { as } m \longrightarrow \infty .
\end{aligned}
$$


Let $k_{0}$ be the positive integer $\max \left(n_{0}, m_{0}\right)$. The open ball $D_{r_{w}}\left(N_{k_{0}}(w)\right)$ around $N_{k_{0}}(w)$ with $r_{w}:=\min \left(\chi\left(N_{k_{0}}(w), \partial D_{\zeta}\right),\left(\frac{1-\lambda_{k_{0}}}{1+\lambda_{k_{0}}}\right) \frac{\chi\left(N_{k_{0}}(w), \zeta\right)}{10}\right)$ has the property

$$
\begin{gathered}
\left(N_{P_{n}, h_{n}} \circ \cdots \circ N_{P_{k_{0}+1}, h_{k_{0}+1}}\right)\left(D_{r_{w}}\left(N_{k_{0}}(w)\right)\right) \cap\left(N_{P_{m}, h_{m}} \circ \cdots \circ\right. \\
\left.N_{P_{k_{0}+1}, h_{k_{0}+1}}\right)\left(D_{r_{w}}\left(N_{k_{0}}(w)\right)\right)=\emptyset
\end{gathered}
$$

for every $m, n>k_{0}, m \neq n$. On the other hand, the rational functions $N_{P_{i}, h_{i}}, 1 \leq i \leq k_{0}$, are in the $\varepsilon_{2}$-neighborhood of $N_{P_{0}, h_{0}}$. Since the points $w$, $N_{P_{0}, h_{0}}(w), \cdots, N_{P_{0}, h_{0}}^{k_{0}}(w)$ are mutually distinct, the points $N_{i}(w)\left(1 \leq i \leq k_{0}\right)$ are mutually distinct, too ( $\varepsilon_{2}$ can be chosen suitably small, if necessary). Therefore there are open neighborhoods $U_{i}$ at $N_{i}(w), 1 \leq i \leq k_{0}-1$, which are mutually disjoint and have empty intersection with $D_{\zeta}$. Now we consider the open neighborhood $\left(N_{k_{0}}\right)^{-1}\left(D_{r_{w}}\left(N_{k_{0}}(w)\right)\right) \cap\left(N_{1}\right)^{-1}\left(U_{1}\right) \cap$ $\cdots \cap\left(N_{k_{0}-1}\right)^{-1}\left(U_{k_{0}-1}\right) \cap V_{z}$. This is an open neighborhood of $w$ in $U_{z}$ which "wanders" (Definition 1, sec. 3.2.3)!

If $\zeta$ is an attracting periodic point of period $s$ of the family $\left\{N_{P_{0}, h_{0}}^{n}\right\}$, then we work with mutually disjoint neighborhoods $D_{\zeta_{j}}$ 's, where $\left\{\zeta_{1}=\zeta, \cdots, \zeta_{s}=\right.$ $\left.N_{P_{0}, h_{0}}^{s-1}(\zeta)\right\}$, which play the same as of $D_{\zeta}$ mentioned above. Since $U_{z}$ and $w$ were arbitrary, we have $\mathbf{J} \subset \partial \mathcal{W}$.

Theorem 7 For almost all points z (with respect to the Lebesgue measure on the plane) in $\overline{\mathcal{W}} \backslash\{\infty\}$ :

$$
\lim _{m \rightarrow \infty} r_{m}(z)=0
$$

The proof of this theorem is similar to the standard one. As mentioned in Lyubich 1983, we need three lemmas for the proof of this theorem, which we state again since they are a little bit different in the form from the ones in Lyubich 1983 . 
The first lemma comes from the Koebe's Distortion Theorem (Theorem 7, sec. 2.1.2): since $\left.N_{m}\right|_{D_{r_{m}(z)}(z)}$ is univalent on $D_{r_{m}}(z)$ for every $z$ not belonging to a finite set of critical points with at most $2 m(d-1)$ elements, $\left\{z_{1}, \cdots, z_{2 m(d-1)}\right\}$, we have:

Lemma 8 Let $0<\alpha<1$. There exists a positive number $M(\alpha)$ such that for any two measurable subsets $Y_{1}, Y_{2}$ in $D_{\alpha r_{m}}(z), z \notin\left\{z_{1}, \cdots, z_{2 m(d-1)}\right\}$ :

$$
\frac{\mu\left(N_{m}\left(Y_{1}\right)\right)}{\mu\left(N_{m}\left(Y_{2}\right)\right)} \leq M(\alpha) \frac{\mu\left(Y_{1}\right)}{\mu\left(Y_{2}\right)}
$$

As we know, each $x \in \mathcal{W}$ has a wandering open neighborhood $U_{x}$. Therefore for every positive number $\delta>0$, the set $V_{x}:=U_{x} \cap D_{\delta}(x)$ defines a wandering open neighborhood with diameter less than $\delta$. We consider the covering $\left(\bigcup_{x \in \mathcal{W}} V_{x}\right) \cup\left(\bigcup_{x \in \partial \mathcal{W}} D_{\delta}(x)\right)$ of the compact closed set $\overline{\mathcal{W}}$. There is a Lebesgue number $\eta$ for this covering ([Munkres]). If we denote the positive number $\min \left(\eta, \frac{\delta}{3}\right)$ by $\xi(\delta)$, then we have the next

Lemma 9 For any $\delta>0$ there is a $\xi(\delta)>0$ and a finite set of points $Y_{\delta}$ such that if $z \in \overline{\mathcal{W}}$, then the disc $D_{\delta}(z)$ contains a wandering disc $D_{\xi(\delta)}(w)$ centered at some point $w \in Y_{\delta}$. Lyubich 1983

Notation 4 Let $\epsilon>0$ be given. Denote $\Gamma_{m}(\epsilon):=\overline{\mathcal{W}} \backslash D_{\epsilon}\left(Z_{m}\right)$ and $X_{m}(\epsilon):=$ $N_{m}\left(\Gamma_{m}(\epsilon)\right)$.

Lemma $10 \sum_{m=1}^{\infty} \mu\left(X_{m}(\epsilon)\right)<\infty$. Lyubich 1983

\section{Proof.}

For $\delta=\frac{\epsilon}{3}$ we find a finite set $Y_{\delta}^{\prime} \subset \Gamma_{m}(2 \delta)$ so that $Y_{\delta}^{\prime} \subset Y_{\delta}$ and $\Gamma_{m}(\epsilon) \subset$ $D_{\delta}\left(Y_{\delta}^{\prime}\right)$. By Lemma 8, for every $y \in Y_{\delta}^{\prime}$ : 


$$
\frac{\mu\left(N_{m}\left(D_{\delta}(y)\right)\right)}{\mu\left(N_{m}\left(D_{\xi(\delta)}(y)\right)\right)} \leq M\left(\frac{1}{2}\right) \frac{\mu\left(D_{\delta}(y)\right)}{\mu\left(D_{\xi(\delta)}(y)\right)} \equiv C(\delta)
$$

According to the subadditivity of the measure,

$$
\mu\left(X_{m}(\epsilon)\right) \leq C(\delta) \sum_{y \in Y_{\delta}^{\prime}} \mu\left(N_{m}\left(D_{\xi(\delta)}(y)\right)\right) \leq C(\delta) \sum_{y \in Y_{\delta}} \mu\left(N_{m}\left(D_{\xi(\delta)}(y)\right)\right)
$$

Note that $Y_{\delta}$ is a finite set. Again since $D_{\xi(\delta)}(y)$ is a wandering disc, we have

$$
\sum_{m=1}^{\infty} \mu\left(N_{m}\left(D_{\xi(\delta)}(y)\right)\right)=\mu\left(\bigcup_{m=1}^{\infty} N_{m}\left(D_{\xi(\delta)}(y)\right)\right)<\infty
$$

\section{Proof of Theorem 7.}

Let $X(\epsilon)=\varlimsup_{m \rightarrow \infty} X_{m}(\epsilon)$. Then $X(\epsilon)=\{z \mid z$ is a point in $\overline{\mathcal{W}}$ with $\left.\overline{\lim }_{m \rightarrow \infty} r_{m}(z)>\epsilon\right\}$. From the last lemma, $\mu(X(\epsilon))=0$.

From Theorem 7 we see that the orbits of the critical points converge to the contracting values in $\mathbf{F}$. Since the points on $\mathbf{J}$ do not have this property, and $\mathbf{J} \subset \partial \mathcal{W}$, we deduce again from Theorem 7 that $\mu(\mathbf{J})=0$. 


\subsection{A General Convergent Sequence}

\subsubsection{Introduction}

Let $\left\{P_{n}\right\}_{n \in \mathbf{N}}$ be a sequence of polynomials of the same degree of $P_{0}$ which converges to $P_{0},\left\{h_{n}\right\}_{n \in \mathbf{N}}$ be a sequence of positive real numbers which converges to some $h_{0}>0$, and $k$ be as in the Theorem 4 (sec. 3.2.3).

There are two families to be considered:

the main family:

$$
\mathbf{A}=\left\{N_{1}=N_{P_{1}, h_{1}}, \cdots, N_{n}=N_{P_{n}, h_{n}} \circ \cdots \circ N_{P_{1}, h_{1}}, \cdots\right\}
$$

the truncated family:

$$
\mathbf{B}=\left\{M_{1}=N_{P_{k+1}, h_{k+1}}, \cdots, M_{n}=N_{P_{k+n}, h_{k+n}} \circ \cdots \circ N_{P_{k+1}, h_{k+1}}, \cdots\right\} .
$$

The Julia set $\mathbf{J}(\mathbf{B})$ and Fatou set $\mathbf{F}(\mathbf{B})$ of the family $\mathbf{B}$ have been already studied. The main goal is now to study these two sets for the new family A. The norm on $\overline{\mathbf{C}}$ is the spherical norm.

\subsubsection{The Fatou Set}

The first thing to be considered is the relation between two families: 
Lemma 11 For every compact subset $K$ of a stable domain of the Fatou set $\mathbf{F}(\mathbf{A}), N_{k}(K) \subset \mathbf{F}(\mathbf{B})$, where $\mathbf{F}(\mathbf{B})$ is the Fatou set of the family $\mathbf{B}$.

\section{Proof.}

For every $x_{0}$ in $\mathrm{K}$ and for every $\epsilon^{\prime}>0$ there is some $\delta^{\prime}>0$ such that if $\left|x-x_{0}\right|<\delta^{\prime}$, then $\left|N_{n}(x)-N_{n}\left(x_{0}\right)\right|<\epsilon^{\prime}$, for every integer $\mathrm{n}$. We show that for every $\epsilon>0$ there is some $\delta>0$ such that if $\left|N_{k}\left(x_{0}\right)-y\right|<\delta$, then

$$
\left|M_{j}\left(N_{k}\left(x_{0}\right)\right)-M_{j}(y)\right|<\epsilon, \quad \forall j \in \mathbf{N} .
$$

Given $\epsilon>0$, there is some $\delta_{1}>0$ such that

$$
\left|x-x_{0}\right|<\delta_{1} \Longrightarrow\left\{\begin{array}{l}
\left|N_{k}(x)-N_{k}\left(x_{0}\right)\right|<\epsilon \\
\left|M_{j}\left(N_{k}(x)\right)-M_{j}\left(N_{k}\left(x_{0}\right)\right)\right|<\epsilon
\end{array}\right.
$$

Since $N_{k}\left(U_{\delta_{1}}\left(x_{0}\right)\right)$ is open, there is some $\delta$ such that $U_{\delta}\left(N_{k}\left(x_{0}\right)\right) \subset N_{k}\left(U_{\delta_{1}}\left(x_{0}\right)\right)$. Hence for every $\mathrm{y}$ in $U_{\delta}\left(N_{k}\left(x_{0}\right)\right)$ there is some $\mathrm{x}$ in $U_{\delta_{1}}\left(x_{0}\right)$ with $y=N_{k}(x)$. Then for every $\mathrm{j}$

$$
\left|M_{j}\left(N_{k}(x)\right)-M_{j}\left(N_{k}\left(x_{0}\right)\right)\right|<\epsilon
$$

Thus we have found a $\delta$ for the given $\epsilon$, which means that $\left\{M_{j}\right\}_{j \in \mathbf{N}}$ is equicontinuous on $N_{k}(K)$.

Therefore

$$
N_{k}(K) \subset \mathbf{F}(\mathbf{B}) \text {. }
$$

Lemma 12 For every compact subset $K$ of a stable domain of $\mathbf{F}(\mathbf{B}), N_{k}^{-1}(K) \subset$ $\mathbf{F}(\mathbf{A})$ (for every branch $N_{k}^{-1}$ ).

Proof.

For every $x_{0}$ in $\mathrm{K}$ we have 
$\forall \epsilon>0 \exists \delta^{\prime}>0$ such that $\forall n \in \mathbf{N},\left|x-x_{0}\right|<\delta^{\prime} \Longrightarrow\left|M_{n}(x)-M_{n}\left(x_{0}\right)\right|<\epsilon$.

But $N_{k}$ is a rational function, therefore it satisfies a Lipschitz condition ([Beardon]):

$$
\left|N_{k}(x)-N_{k}\left(x_{0}\right)\right| \leq L\left|x-x_{0}\right|
$$

Thus if $\delta$ is so that $L \delta<\delta^{\prime}$, then for each $\mathrm{x}$ in $U_{\delta}\left(N_{k}^{-1}\left(x_{0}\right)\right)$ we have:

$$
\left|N_{k}(x)-x_{0}\right|=\left|N_{k}(x)-N_{k}\left(N_{k}^{-1}\left(x_{0}\right)\right)\right| \leq L\left|x-N_{k}^{-1}\left(x_{0}\right)\right|<L \delta<\delta^{\prime} .
$$

Therefore

$$
\begin{gathered}
\left|N_{n}(x)-N_{n}\left(N_{k}^{-1}\left(x_{0}\right)\right)\right|= \\
M_{n}\left(N_{k}(x)\right)-M_{n}\left(N_{k}\left(N_{k}^{-1}\left(x_{0}\right)\right)\right) \mid \\
<\epsilon .
\end{gathered}
$$

Hence

$$
N_{k}^{-1}(K) \subset \mathbf{F}(\mathbf{A})
$$

Now consider a stable domain $\mathrm{V}$ of $\mathbf{F}(\mathbf{A})$. For every integer $\mathrm{s}$, every $\mathrm{x}$ and $\mathrm{y}$ in $\mathrm{V}$ and every compact subset $\mathrm{K}$ of $\mathrm{V}$ containing $\mathrm{x}$ and $\mathrm{y}$ we deduce

$$
N_{s}(K) \subset \mathbf{F}(\mathbf{B})
$$

for every n greater than s. From the previous results, the stable domain V is actually a contracting domain, hence of the form $B^{\prime}\left(\zeta_{i}\right)$, where $P_{0}\left(\zeta_{i}\right)=0$ and $B^{\prime}\left(\zeta_{i}\right)=\left\{z \in \mathbf{C} \mid N_{n}(z) \longrightarrow \zeta_{i}\right.$ as $\left.n \longrightarrow \infty\right\}$. It yields that 


$$
\mathbf{F}(\mathbf{A})=\bigcup_{P_{0}\left(\zeta_{i}\right)=0} B^{\prime}\left(\zeta_{i}\right)
$$

\subsubsection{The Julia Set}

The Julia set $\mathbf{J}(\mathbf{A})$ is by far a nonempty, closed, nowhere dense and perfect subset of $\overline{\mathbf{C}}$.

Let $x_{0}$ be in $\mathbf{J}(\mathbf{A})$. If $N_{s}\left(x_{0}\right) \in \mathbf{F}(\mathbf{B})$ for some positive integer $s$, then a subsequence $\left\{M_{n_{k}}\right\}$ of $\left\{M_{n}\right\}$ converges uniformly to a $P_{0}$-root $\zeta$ on a neighborhood of $N_{s}\left(x_{0}\right)$ as k goes to infinity. Since $N_{s}$ is a rational map, it is an open map which means that a neighborhood of $N_{s}\left(x_{0}\right)$ can be considered as an image $N_{s}(U)$ of an open connected neighborhood of $x_{0}$. Thus $\left\{M_{n_{k}} \circ N_{s}\right\}_{k \in \mathbf{N}}$ is uniformly convergent on $\mathrm{U}$. The family $\left\{M_{n_{k}} \circ N_{s}\right\}_{k \in \mathbf{N}}$ defines a subsequence $\left\{N_{n_{k}}\right\}$ of $\left\{N_{n}\right\}$ on U, i.e. $\left\{N_{n}\right\}$ is equicontinuous on $\mathrm{U}$, therefore normal at $x_{0}$ which contradicts our assumption. Hence

$$
N_{s}(\mathbf{J}(\mathbf{A})) \subset \mathbf{J}(\mathbf{B}) .
$$

Indeed, $N_{s}(\mathbf{J}(\mathbf{A}))=\mathbf{J}(\mathbf{B})$, since for every stable domain $\mathrm{V}$ of $\mathbf{F}(\mathbf{B})$ there is some stable domain $\mathrm{U}$ of $\mathbf{F}(\mathbf{A})$ such that

$$
N_{s}(U)=V
$$

and vice versa. Therefore the connected components of $N_{s}^{-1}(V)$ are stable domains of $\mathbf{F}(\mathbf{A})$, too. Hence for every stable domain $\mathrm{V}$ of $\mathbf{F}(\mathbf{B})$, 
$\partial V=N_{s}(\partial U)$

Thus

$$
\text { (2) } \quad \mathbf{J}(\mathbf{B}) \subset N_{s}(\mathbf{J}(\mathbf{A})) \text {. }
$$

From $(1,2)$ we have

$$
N_{s}^{-1}(\mathbf{J}(\mathbf{B}))=\mathbf{J}(\mathbf{A})
$$

Thus $\mathbf{J}(\mathbf{A})$ has no isolated points, too, and is therefore perfect.

\subsubsection{Connectedness of the Julia Set}

To study the behavior of the Julia set of the family $\left\{N_{n}\right\}$ with respect to the Julia set of the family $\left\{N_{P_{0}, h_{0}}^{n}\right\}$ as $\left(P_{n}, h_{n}\right)$ converges to $\left(P_{0}, h_{0}\right)$, we need a distance on the set of all nonempty, compact subsets of the complex sphere $\overline{\mathrm{C}}$ :

Definition 3 Consider the complex sphere $\overline{\mathbf{C}}$ to be equipped with the spherical metric. Let $\epsilon$ be a positive number and $A \subset \overline{\mathbf{C}}$. We define the set $U_{\epsilon}(A)$ as follows:

$$
U_{\epsilon}(A)=\bigcup_{a \in A} D_{\epsilon}(a)
$$

We denote the collection of all nonempty compact subsets of $\overline{\mathbf{C}}$ by $\mathcal{H}$. 
Definition 4 For the elements $A, B \in \mathcal{H}$ we define

$$
D(A, B)=\sup \left\{\epsilon>0 \mid A \subset U_{\epsilon}(B) \text { and } B \subset U_{\epsilon}(A)\right\}
$$

The function $D: \mathcal{H} \times \mathcal{H} \longrightarrow \mathbf{R}^{+}$defines a distance known as Hausdorff distance on $\mathcal{H}$ so that the space $\mathcal{H}$ with the metric induced by $D$ is a compact metric space [Munkres].

Theorem 8 Assume the sequence of polynomials $\left\{P_{n}\right\}_{n \in \mathbf{N}}$ converges to the fixed polynomial $P_{0}$ with respect to the uniform metric, and $\left\{h_{n}\right\}$ converges to $h_{0} \in(0,1]$, as $n$ goes to $\infty$. Also assume the Euler's map $N_{P_{0}, h_{0}}$ is subhyperbolic as before. Then

$$
\lim _{n \rightarrow \infty} N_{n}^{-1}\left(\mathbf{J}\left(N_{P_{0}, h_{0}}\right)\right)=\mathbf{J}
$$

with respect to Hausdorff metric (we recall that $\mathbf{J}$ is the Julia set of the family $\left\{N_{n}\right\}$ ).

\section{Proof.}

We know already from Lemma 5, sec. 3.3.2 that for every $\varepsilon>0$ there is some $m>0$ such that

$$
\mathbf{J}(\mathbf{A}) \subset U_{\varepsilon}\left(\mathbf{J}\left(N_{P_{0}, h_{0}}\right)\right)
$$

where $\mathrm{A}=\left\{N_{m+n}\right\}_{n \in \mathbf{N}}$. But $N_{m}^{-1}(\mathbf{J}(\mathbf{A}))=\mathbf{J}$, thus

$$
N_{m}(\mathbf{J}) \subset U_{\varepsilon}\left(\mathbf{J}\left(N_{P_{0}, h_{0}}\right)\right) .
$$

Second, we will show that for every $\varepsilon>0$ there is some integer $\mathrm{m}$ such that for every $n>m$ :

$$
N_{n}^{-1}\left(\mathbf{J}\left(N_{P_{0}, h_{0}}\right)\right) \subset U_{\varepsilon}(\mathbf{J}) .
$$


If this is not true, there is a sequence $\left\{z_{n}\right\} \subset \overline{\mathbf{C}}$ outside an $\varepsilon$-neighborhood $U_{\varepsilon}(\mathbf{J})$ of $\mathbf{J}$ such that

$$
N_{n}\left(z_{n}\right)=\zeta_{n}, \text { where } \zeta_{n} \in \mathbf{J}\left(N_{P_{0}, h_{0}}\right)
$$

Since $\left\{z_{n}\right\}$ lies in the compact set $S:=\overline{\mathbf{C}} \backslash U_{\varepsilon}(\mathbf{J})$, it has a subsequence $\left\{z_{\lambda_{n}}\right\}$ which converges to $z_{0} \in S$ as $\mathrm{n}$ goes to $\infty$. This yields $z_{0} \in \mathbf{F}$. Thus $\left\{N_{n}\right\}$ is normal in a neighborhood $V_{z_{0}}$ of $z_{0}$. Hence the subsequence $\left\{N_{\lambda_{n}}\right\}$ has again a convergent subsequence denoted as $\left\{N_{s}\right\}$ such that

$$
N_{s}(z) \longrightarrow G(z)=G\left(z_{0}\right) \quad \text { as } s \longrightarrow \infty,
$$

locally uniformly on $V_{z_{0}}$ for some meromorphic function G. Specially, $N_{s}\left(z_{s}\right) \longrightarrow G\left(z_{0}\right)$, as $s \longrightarrow \infty$. But $N_{s}\left(z_{s}\right)=\zeta_{s} \in \mathbf{J}\left(N_{P_{0}, h_{0}}\right)$ which is a closed set. Therefore

$$
G\left(z_{0}\right):=\zeta \in \mathbf{J}\left(N_{P_{0}, h_{0}}\right) .
$$

So it yields that $N_{s}\left(z_{0}\right)$ goes to $\zeta \in \mathbf{J}\left(N_{P_{0}, h_{0}}\right)$, while $z_{0} \in \mathbf{F}$. But it contradicts the fact that every such $\zeta$ belongs to $\mathbf{F}\left(N_{P_{0}, h_{0}}\right)$.

$N_{P_{0}, h_{0}}$ is subhyperbolic. Therefore its Julia set $\mathbf{J}\left(N_{P_{0}, h_{0}}\right)$ is a (connected,) locally connected closed set in $\overline{\mathbf{C}}$ [Milnor, chap. 19]. On the other hand, the Hausdorff limit of connected sets is connected, too. Thus

Theorem 9 The Julia set $\mathbf{J}$ is connected.

Corollary $\mathbf{2}$ The Fatou set $\mathbf{F}$ has simply connected components.

Indeed, we can prove a stronger result, namely

Theorem $10 \mathbf{J}$ is locally connected. 


\section{Proof.}

The set $\mathbf{J}\left(N_{P_{0}, h_{0}}\right)$ is locally cannected, that is each point $z \in \mathbf{J}\left(N_{P_{0}, h_{0}}\right)$ has an open neighborhood $U_{z}$ which is connected. Since $\mathbf{J}\left(N_{P_{0}, h_{0}}\right)$ is a compact set, the covering $\left\{U_{z}\right\}$ has a finite subcovering $\left\{U_{1}, \cdots, U_{p}\right\}$ such that $\mathbf{J}\left(N_{P_{0}, h_{0}}\right) \subset \bigcup_{j=1}^{p} U_{i}$. If we let $\lambda:=\min \left\{\operatorname{diam}\left(U_{j}\right) \mid 1 \leq j \leq p\right\}$, where $\operatorname{diam}\left(U_{j}\right)$ is the diameter of the set $U_{j}$ in spherical metric, then $0<\lambda<\infty$. According to Theorem 8, there is some $m_{0}$ such that $N_{m_{0}}(\mathbf{J}) \subset$ $U_{\frac{\lambda}{2}}\left(\mathbf{J}\left(N_{P_{0}, h_{0}}\right)\right)$. So each point $w \in \mathbf{J}$ maps by $N_{m_{0}}$ to some $U_{j}$. Therefore the connected component of $N_{m_{0}}^{-1}\left(U_{j}\right)$ which contains $w$ is a connected open neighborhood of $w$. 


\section{An Application: the Newton's Petals}

\subsection{Basic Assumptions}

We begin this section with the following assumptions on the relaxed Newton's function $N_{P_{0}, h_{0}}$ introduced in section 3.1:

$1^{\prime}$. The polynomial $P_{0}$ with $\operatorname{deg} P_{0}=d \geq 2$ has at least two different roots, and $h_{0} \in(0,1]$, as before (sec. 3.1);

$2^{\prime} . N_{P_{0}, h_{0}}$ has just one parabolic cycle $\left\{0, N_{P_{0}, h_{0}}(0)=\zeta_{1}, \cdots, N_{P_{0}, h_{0}}^{m-1}(0)=\right.$ $\left.\zeta_{m-1}\right\}$ assumed to contain the origin (without loss of generality, since otherwise we can map this point to 0 by a suitable conjugacy) of length (period) $m$ for some positive integer $m>1$, and multiplier $\left(N^{m}\right)_{P_{0}, h_{0}}^{\prime}(0)=\lambda$ for which there is some positive integer $q$ with $\lambda^{q}=1$.

The first assumption is the same as "assumption 1" in sec. 3.1. From the second one we deduce that the forward orbit of 0 under the function $N_{P_{0}, h_{0}}$ is the finite set $\left\{0, N_{P_{0}, h_{0}}(0)=\zeta_{1}, \cdots, N_{P_{0}, h_{0}}^{m-1}(0)=\zeta_{m-1}\right\}$, where all of $\zeta_{i}$ 's are in $\mathbf{C}$. We already know from section 2.2 that $\left\{0, \zeta_{1}, \cdots, \zeta_{m-1}\right\} \subset \mathbf{J}\left(N_{P_{0}, h_{0}}\right)$, and the rational function $N_{P_{0}, h_{0}}^{m}$ has the following form (if necessary, after applying a conjugacy) in a neighborhood of the origin which is now a fixed point of $N_{P_{0}, h_{0}}^{m}$ :

$$
N_{P_{0}, h_{0}}^{m}(z)=\lambda z-z^{s+1}+O\left(z^{s+2}\right)
$$

where $s=l q$, for some positive integer $l$.

Hence there are $s$ petals at $z=0$ for $N_{P_{0}, h_{0}}^{m}$, these dividing into $l$ sets of $q$ petals such that $N_{P_{0}, h_{0}}^{m}$ acts as a cycle of length $q$ on each such set, i. e. each such set is invariant under $N_{P_{0}, h_{0}}^{m}$. 
Theoretically, these $s$ petals are defined by

$$
\Pi_{k}(t)=\left\{r e^{i \theta}\left|r^{s}<t(1+\cos (s \theta)) \leq 2 t ;\right| 2 k \pi / s-\theta \mid<\pi / s\right\},
$$

for some suitable positive number $t$. We may define $\epsilon=1 / t$ and write the above formula in the form

$$
\Pi_{k}(t)=\left\{r e^{i \theta}\left|\epsilon^{1 / s} r<(1+\cos (s \theta))^{1 / s} ;\right| 2 k \pi / s-\theta \mid<\pi / s\right\}
$$

which we use in section 4.2.2.

In practice, one can only trace the petals with approximating methods. One of these methods is based on the fact that a rationally indifferent fixed point can be considered as the limit of the attracting case, as we move the attracting fixed point in its immediate attracting basin $U$ to the boundary $\partial U \subset \mathbf{J}\left(N_{P_{0}, h_{0}}\right)$ [Beardon. The important point in this process is that our movements are not any more defined by the isometries of the plane domains which are conformal transformations, but by the quasiconformal ones. We develop this approach in the next paragraphs for petals $\Pi_{k}$ of the function $N_{P_{0}, h_{0}}$ mentioned above by means of the subhyperbolic approximations produced by a method known as "quasiconformal surgery" . 


\subsection{Virtual Petals}

\subsubsection{Quasiconformal Mappings}

Let $f: D \longrightarrow D$ be any function with continuous partial derivatives $f_{x}=$ $\partial f / \partial x$ and $f_{y}=\partial f / \partial y$ in a domain $D \subset \overline{\mathbf{C}}$. We first fix the notations

$$
d z=d x+i d y \quad, \quad d \bar{z}=d x-i d y
$$

and then introduce the two differential operators

$$
\begin{array}{r}
\partial f / \partial \bar{z}=\frac{1}{2}(\partial f / \partial x+i \partial f / \partial y) \\
\partial f / \partial z=\frac{1}{2 i}(\partial f / \partial x-i \partial f / \partial y)
\end{array}
$$

Following the above terminology, we denote $\partial f / \partial \bar{z}$ by $f_{\bar{z}}$ and $\partial f / \partial z$ by $f_{z}$. We see also that the Jacobian $J_{f}$ of $f$ is given by

$$
J_{f}=\left|f_{z}\right|^{2}-\left|f_{\bar{z}}\right|^{2}
$$

which shows that $f$ preserves orientation if and only if $\left|f_{\bar{z}}\right|<\left|f_{z}\right|$.

If $f$ is an analytic function, $i$. e. it is derivable with respect to the complex variable $z$, then according to the Cauchy-Riemann equations for analytic functions we have

$$
\partial f / \partial \bar{z}=0 \quad \text { and } \quad \partial f / \partial z=f^{\prime}(z) .
$$

Therefore, $f_{\bar{z}} / f_{z}=0$. For a general function $f$ with partial derivatives

$f_{x}, f_{y}$ in the domain $D$ we can generalize the Cauchy-Riemann equations to the Beltrami equation (Carleson-Gamelin, chap. I, sec. 5], [Beardon, sec. 8.3]): 


$$
\left(^{*}\right) \quad f_{\bar{z}} / f_{z}=\mu,
$$

where $\mu: D \longrightarrow \mathbf{C}$ is a Lebesgue measurable function with $\|\mu\|_{\infty}<1$ in $D$, and so $|\mu| \leq\|\mu\|_{\infty}$ everywhere in $D$ except on a set of Lebesgue measure zero. A function $\mu$ with these properties is called a Beltrami coefficient. The norm |.| comes from the metric $\rho$ defined in sec. 2.1.2.

Definition 1 Given a domain $D$ and a Beltrami coefficient $\mu$ on $D$, we say that a homeomorphism $f$ on $D$ is quasiconformal with complex dilatation $\mu$ in $D$ if $f$ is a $\mathcal{L}^{2}$-solution of $\left(^{*}\right)$ in $D$ ([Beardon, p. 180], [Lehto, p. 24], Ahlfors, chap. I]).

We always assume that the quasiconformal function $f$ is an orientationpreserving diffeomorphism, which means that $\mu$ is a continuous function with $|\mu|<1$ for such $f$.

If we let $f$ be represented in the form $w=f(z)=f(x+i y)=u(x+i y)+$ $i v(x+i y)$, then $d w=d u+i d v=f_{z} d z+f_{\bar{z}} d \bar{z}$. Therefore:

$$
d u^{2}+d v^{2}=\left(u_{x}^{2}+v_{x}^{2}\right) d x^{2}+2\left(u_{x} u_{y}+v_{x} v_{y}\right) d x d y+\left(u_{y}^{2}+v_{y}^{2}\right) d y^{2}
$$

This yields that $d f$ maps an infinitesimal ellipse in the tangent plane at $z$ with the generators $(d x, d y)$ to a circle in the tangent plane at $f(z)$ with the generators $(d u, d v)$. Since

$$
\left(\left|f_{z}\right|-\left|f_{\bar{z}}\right|\right)|d z| \leq|d w| \leq\left(\left|f_{z}\right|+\left|f_{\bar{z}}\right|\right)|d z|
$$

we see that the ratio of the major axis of this infinitesimal ellipse to its minor axis is

$$
D_{f}=\frac{\left|f_{z}\right|+\left|f_{\bar{z}}\right|}{\left|f_{z}\right|-\left|f_{\bar{z}}\right|} \geq 1
$$


Definition 2 This ratio $D_{f}$ is called the dilatation of $f$ at the point $z$ in the domain D. The quasiconformal map $f$ with $D_{f} \leq K<\infty$ in $D$ is said to be K-quasiconformal in D. Ahlfors

The argument of the minor axis of the infinitesimal ellipse (in the tangent plane with generators $(d x, d y)$ ), which is indeed the argument of $d z$ ( $d z$ and $d \bar{z}$ are not perpendicular: they make an angle of $\arg \left(\mu_{f}\right)$ at the origin), is $\arg \left(\mu_{f}\right) / 2$, where $\mu_{f}$ is the complex dilatation of $f$. Therefore the argument of the major axis is $\arg \left(\mu_{f}\right) / 2+\pi / 2$. Carleson-Gamelin, p. 16], Ahlfors, chap. 1]

The relation between the comlex dilatation $\mu_{f}$ of a quasiconformal map $f$ and its dilatation $D_{f}$ is [Ahlfors, chap. 1]

$$
D_{f}=\frac{1+\left|\mu_{f}\right|}{1-\mid \mu_{f}} .
$$

Therefore, we can assign some $0 \leq k<1$ to each $K$-quasiconformal function $f(0 \leq K<\infty)$ so that $\left|\mu_{f}\right|<k$ in the domain $D$, and call $f$ $k$-quasiconformal as well. For most applications, we take $\overline{\mathbf{C}}$ as $D$ and just say $f$ is quasiconformal.

In order to have a quasiconformal function on a domain, we need to show that the solutions of $(*)$ do exist and are unique [Beardon, p. 180]:

Theorem 1 Let $\mu$ be a Beltrami coefficient on a domain D on the the sphere. Then:

1. there exists a quasiconformal map with complex dilatation $\mu$ almost everywhere (with respect to the Lebesgue measure on the sphere) on $D$ Lehto, Theorem 4.4, p. 28]; and

2. if $\phi$ and $\psi$ are two such maps, then $\phi \psi^{-1}$ is conformal (hence analytic) [Lehto, Theorem 4.2, p. 24]. 
There is also another important theorem known as Measurable Riemann Mapping Theorem [Beardon, p. 181]:

Theorem 2 (Measurable Riemann Mapping Theorem) Given a simply connected domain $D$ (conformally equivalent to the unit disc $\Delta=\{z \mid$ $\chi(0, z)<1\})$, and a Beltrami coefficient $\mu$ on $D$, there is a quasiconformal map $\phi$ of $D$ onto $\Delta$ with the complex dilatation $\mu$ almost everywhere (with respect to Lebesgue measure on the sphere) in D.

The case $\mu \equiv 0$ is the classical Riemann Mapping Theorem.

\subsubsection{The Process of Approximating}

As mentioned in the introduction to this chapter, one can purterb a rational function $f: \overline{\mathbf{C}} \longrightarrow \overline{\mathbf{C}}$ with the help of the quasiconformal surgery to make all of its nonrepelling periodic points attracting. We explain this so-called surgery by applying a method used in the proof of Theorem 1 in Shishikura 1987] to our special case explained in sec. 4.1. Let us assume the rational function $f$ to be $N_{P_{0}, h_{0}}(z)=z-h_{0} P_{0}(z) / P_{0}^{\prime}(z)$. According to assumption $2^{\prime}$ it has one rationally indifferent periodic point at $z=0$ with period $m$ among its nonrepelling periodic points, the others being all (super-) attracting periodic points. Thus we have

$$
N_{P_{0}, h_{0}}^{m}(z)=\lambda z-z^{s+1}+O\left(z^{s+2}\right)
$$


in a neighborhood of 0 , where $\lambda$ is the multiplier $\left(N^{m}\right)_{P_{0}, h_{0}}^{\prime}(0)$ for which there is some positive integer $q$ with $\lambda^{q}=1$, and $s=l q$ as mentioned in sec. 4.1. If we define

$$
U_{0}^{\prime}=\left\{z|0<| z\left|<r_{0},\right| \arg z^{q} \mid<\pi / 2 l\right\}
$$

for some suitably small $r_{0}>0$, then we have $N_{P_{0}, h_{0}}^{m}\left(\overline{U_{0}^{\prime}}\right) \subset U_{0}^{\prime} \cup\{0\}([$ Beardon, Lemma 6.5.2, p. 111]). Let us consider first some $w_{0} \in N_{P_{0}, h_{0}}^{-1}\left(U_{0}^{\prime} \cup\{0\}\right) \backslash$ $N_{P_{0}, h_{0}}^{m-1}\left(\overline{U_{0}^{\prime}}\right)$ and define the automorphism (holomorphic diffeomorphism of the sphere) $T$ as

$$
T(z)=\frac{w_{0} z}{z-a}
$$

where $a \in \overline{\mathbf{C}}, a \neq w_{0}$ and $a w_{0} \neq 0$. We note that the composition $f=T^{-1} \circ N_{P_{0}, h_{0}} \circ T$ takes $\infty$ to some finite point in $T^{-1}\left(U_{0}^{\prime}\right)$ for suitably small $r_{0}$, and $f^{m}$ has a rational indifferent fixed point at 0 . In order to use the next theorems we first apply the surgery process to $f$ and $T^{-1}\left(U_{0}^{\prime}\right)$ which we denote again by $U_{0}^{\prime}$, and then change the scale.

There is a complex polynomial $h$ such that $h\left(\zeta_{i}\right)=0$ and $h^{\prime}\left(\zeta_{i}\right)=-1$, where $i=0, \cdots, m-1, \zeta_{0}=0$ and $\zeta_{i}=f^{i}(0)$, then

Lemma 1 If $H_{\epsilon}: \overline{\mathbf{C}} \longrightarrow \overline{\mathbf{C}}$ is defined by

$$
\begin{aligned}
& H_{\epsilon}(z)=z+\epsilon h(z) \cdot \eta\left(\epsilon^{1 / m}|z|\right) \quad z \in \mathbf{C}, \epsilon>0 ; \\
& H_{\epsilon}(\infty)=\infty,
\end{aligned}
$$

where $\eta$ is a smooth real function on the set of real numbers $\mathbf{R}$ with values in $[0,1]$ such that $\eta \equiv 1$ on $[0,1]$ and $\eta \equiv 0$ on $[2, \infty)$, then $H_{\epsilon}$ is quasiconformal for small numbers $\epsilon$. Furthermore, $H_{\epsilon} \longrightarrow i d_{\overline{\mathbf{C}}}$ uniformly, and $\left\|\mu_{H_{\epsilon}}\right\|_{\infty} \longrightarrow 0$, as $\epsilon \longrightarrow 0$. [Shishikura 1987, Lemma 2, p. 8-9] 
We consider the function $g_{\epsilon}=f \circ H_{\epsilon}$. For small $\epsilon>0$ as above the function $g_{\epsilon}^{m}$ is equal to $f^{m}$ out of a neighborhood of zero which contains the petals of $f^{m}$.

The function $g_{\epsilon}^{m}$ has a Taylor expantion in a suitably small neighborhood of 0 :

$$
g_{\epsilon}^{m}(z)=\lambda z\left[(1-\epsilon)^{m}-z^{s}+O(\epsilon z)+O\left(z^{s+1}\right)\right]
$$

Shishikura 1987, p. 10]. There is also a subset $U_{\epsilon}^{\prime}=U_{0}^{\prime} \cap\{z \in \mathbf{C}|| z \mid<$ $\left.\epsilon^{r / 2 m-1}\right\}$ of $U_{0}^{\prime}$ such that $g_{\epsilon}^{m}\left(\overline{U_{\epsilon}^{\prime}}\right) \subset U_{\epsilon}^{\prime}$, for suitably small $r>0$ ([Shishikura 1987, p. 10]). Hence the point 0 is an attracting fixed point of $g_{\epsilon}^{m}$, or an attracting periodic point of $g_{\epsilon}$ of period $m$.

The function $g_{\epsilon}$, which is the quasiconformal perturbation of $f$, inherits the attracting periodic points of $f$. It changes the rationally indifferent nature of zero to an attracting one. Furthermore, $g_{\epsilon} \longrightarrow f$ uniformly as $\epsilon \longrightarrow 0$. The function $g_{\epsilon}^{m}$ joins all petals of $f$ at zero to build an open set which is the immediate basin of 0 and has 0 naturally in its interior (Fig. 1; compare also with Figure 1, sec. 2.2).

To make the functions $g_{\epsilon}$ rational we need the following

Lemma 2 Suppose that a polynomial $h(z)$, a rational function $f(z)$ and open sets $E_{\epsilon}$ of $\overline{\mathbf{C}}\left(0 \leq \epsilon<\epsilon_{0}\right)$ satisfy:

a. $E_{0} \subset E_{\epsilon}$, and $E_{\epsilon}$ 's are uniformly bounded sets in $\mathbf{C}$;

b. $f(\infty) \in E_{0}$;

c. $f \circ(i d+\epsilon h)\left(E_{\epsilon}\right) \subset E_{\epsilon}$.

If we set 


$$
\begin{gathered}
H_{\epsilon}(z)=z+\epsilon h(z) \cdot \eta\left(\epsilon^{1 / k}|z|\right) \quad z \in \mathbf{C}, \epsilon>0, \\
H_{\epsilon}(\infty)=\infty,
\end{gathered}
$$

and $g_{\epsilon}=f \circ H_{\epsilon}$, then for small $\epsilon>0$ there exist quasiconformal mappings $\phi_{\epsilon}$ of $\overline{\mathbf{C}}$ such that $f_{\epsilon}=\phi_{\epsilon} \circ g_{\epsilon} \circ \phi_{\epsilon}^{-1}$ are rational functions. Furthermore, $\phi_{\epsilon} \longrightarrow i d_{\overline{\mathbf{C}}}$, and $f_{\epsilon} \longrightarrow f$ as $\epsilon \longrightarrow 0$. [Shishikura 1987, p. 9]

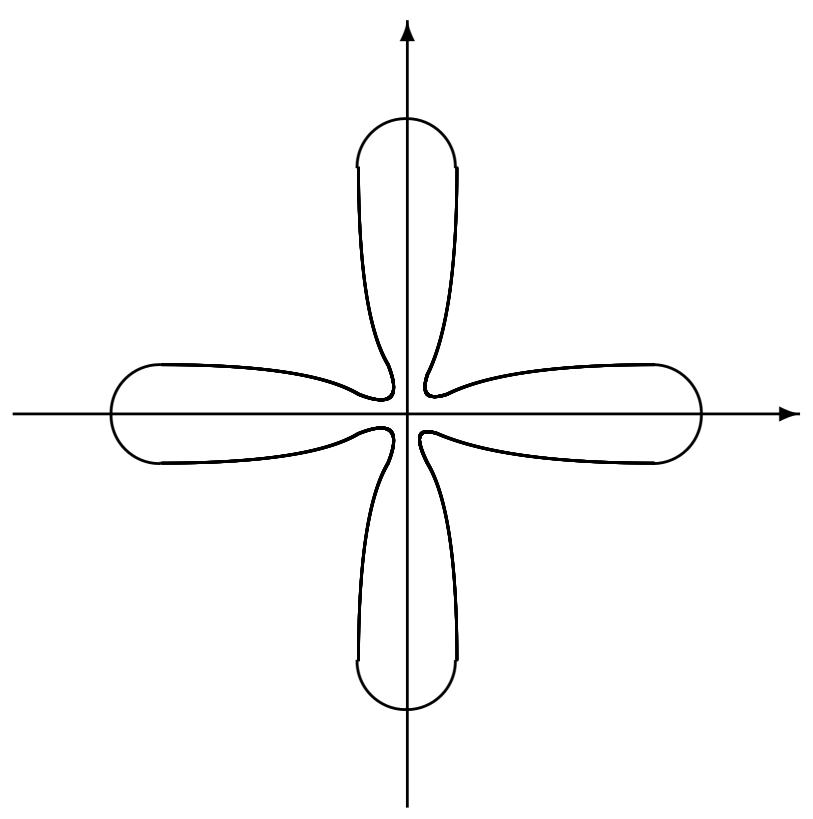

Figure 1

The perturbed domain for $s=4$

First we apply the last lemma to the case where $g_{\epsilon}$ and $f$ are defined as above, and $E_{\epsilon}=U_{\epsilon}^{\prime} \cup g_{\epsilon}\left(U_{\epsilon}^{\prime}\right) \cup \cdots \cup g_{\epsilon}^{m-1}\left(U_{\epsilon}^{\prime}\right)$. The rational functions $f_{\epsilon}$ 
obtained are subhyperbolic (since the composition $f_{\epsilon}=\phi_{\epsilon} \circ g_{\epsilon} \circ \phi_{\epsilon}^{-1}$ does not change the nature of an attracting periodic point) and converge uniformly to $f$. Then we change the scale to obtain the functions $F_{\epsilon} \bar{T} \circ f_{\epsilon} \circ T^{-1}$. Although they still have the origin as an attracting periodic point of order $m$ and are equal to $N_{P_{0}, h_{0}}$ out of a neighborhood of zero, they are not yet our candidates for approximating the petals, since they do not have the form of a relaxed Newton's function. To this purpose we need the following theorem which is a special case of the well known "Runge's Theorem" [Rudin, p. 257]:

Theorem 3 Suppose $K$ is a compact set in the plane, $\overline{\mathbf{C}} \backslash K$ is connected, and $f$ is a function holomorphic in some open set containing $K$. Then there is a sequence $\left\{P_{n}\right\}$ of polynomials such that $P_{n}(z) \longrightarrow f(z)$ uniformly on $K$.

In our case we take the union of the closures of the petals of $N_{P_{0}, h_{0}}$, $\bar{\Pi}_{0} \cup \cdots \cup \bar{\Pi}_{m l q-1}$, as the compact set $K$ in the above theorem. Since $F_{\epsilon}$ is holomorphic for small $\epsilon>0$, we can introduce the holomorphic function

$$
G_{\epsilon}(z)=\exp \left\{\int \frac{h_{0}}{z-F_{\epsilon}(z)} d z\right\} .
$$

According to Theorem 1, for each suitably small $\epsilon>0$ there exist a polynomial $P_{\epsilon}$ and a real number $c_{\epsilon}>0$ such that

$$
\begin{gathered}
\left|P_{\epsilon}(z)-G_{\epsilon}(z)\right|<\epsilon \\
\text { and } \\
\left|N_{P_{\epsilon}, h_{0}}(z)-F_{\epsilon}(z)\right|<\epsilon c_{\epsilon},
\end{gathered}
$$

in a domain $\Omega$ which contains $K$, where the rational function $N_{P_{\epsilon}, h_{0}}$ is the relaxed Newton's function for the pairs $\left(P_{\epsilon}, h_{0}\right)$.

For suitably small $\epsilon$ the function $N_{P_{\epsilon}, h_{0}}$ has an attracting periodic point $z_{\epsilon}$ of order $m$ near 0 with the property $z_{\epsilon} \longrightarrow 0$, as $\epsilon \longrightarrow 0$. Furthermore, $N_{P_{\epsilon}, h_{0}}^{m}\left(\overline{U_{\epsilon}}\right) \subset U_{\epsilon}$. Let us take a strictly decreasing sequence of positive real 
numbers $\left\{\epsilon_{n}\right\}_{n \in \mathbf{N}}$ so that $\epsilon_{n} \longrightarrow 0$ as $n \longrightarrow \infty$, and $N_{P_{\epsilon_{n}}, h_{0}}^{m}\left(\bar{U}_{\epsilon_{n}}\right) \subset \bar{U}_{\epsilon_{n+1}} \subset$ $U_{\epsilon_{n}}$. If we define the functions $N_{n}$ by

$$
N_{n}(z)=\left.\left.N_{P_{\epsilon_{n}}, h_{0}}\right|_{\Omega} \circ \cdots \circ N_{P_{\epsilon_{1}}, h_{0}}\right|_{\Omega}(z)
$$

then we obtain a family $\left\{N_{n}\right\}_{n \in \mathbf{N}}$ already studied in chapter 3 , whose dynamics near zero approximates that of the family $\left\{N_{P_{0}, h_{0}}^{n}\right\}$ near zero. 


\section{References}

\section{References}

[Ahlfors] L. V. Ahlfors: Complex Analysis, McGraw-Hill, Singapore, 1979.

[Ahlfors] L. V. Ahlfors: Lectures on Quasiconformal Mappings, Van Nostrand, Princeton, 1966.

[Beardon] A. F. Beardon: Iteration of Rational Functions, Springer Verlag NewYork, 1991.

[Benzinger] H. E. Benzinger: Plane autonomous systems with rational vector fields, TAMS 326 (1991), 465-484.

[Bueger] M. Bueger: Eine Verallgemeinerung der klassischen Iterationstheorie, Mitteil. Math. Seminar Giessen 224, 1995.

[Carleson-Gamelin] L. Carleson, T. W. Gamelin: Complex Dynamics, Springer Verlag, New York, 1993.

[Eremenko-Lyubich] A. E. Eremenko, M. Yu. Lyubich: The dynamics of analytic transformations, Leningrad Math. J., Vol. 1:3 (1990), 563634.

[Golusin] G. M. Golusin: Geometrische Funktionentheorie, VEB Deutscher Verlag der Wissenschaften, Berlin, 1957.

[Haeseler-Kriete] : The relaxed Newton's method for rational functions, Random and Computational Dynamics 3 (1995), 71-92.

[Henrici] P. Henrici: Discrete Variable Methods in Ordinary Differential Equations, John Wiley \& Sons, New York, 1962. 
[Hubbard-West] J. H. Hubbard, B. H. West: Differential Equations: A Dynamical Systems Approach, Part I, Springer Verlag, New York, 1991.

[Jones-Singerman] G. A. Jones, D. Singerman: Complex Functions: an algebraic and geometric viewpoint, Cambridge University Press, Cambridge, 1987.

[Kriete] H. Kriete: Discrete and continuous complex dynamics, Preprint, Göttingen, 2000.

[Lehto] O. Lehto: Univalent Functions and Teichmuller Spaces, Graduate Texts in Mathematics Vol. 109, Springer Verlag, 1987.

[Lyubich 1983] M. Yu. Lyubich: On the typical behavior of the trajectories of a rational mapping of the sphere, Soviet Math. Dokl. Vol. $27: 1$ (1983), 22-25.

[Lyubich 1986] M. Yu. Lyubich: The dynamics of rational transforms: the topological picture, Russian Math. Surveys 41:4 (1986), 43-117.

[Mañe, Sad, Sullivan] R. Mañe, P. Sad, D. Sullivan: On the dynamics of rational maps, Ann. scient. Éc. Norm. Sup., $4^{e}$ série, t. 16 (1983), 193-217.

[Milnor] J. Milnor: Dynamics in One Complex Variable, Vieweg, 1999.

[Munkres] J. R. Munkres: Topology, A First Course, Prentice-Hall, 1975.

[Perko] L. Perko: Differential Equations and Dynamical Systems, Springer Verlag, 1991.

[Rudin] W. Rudin: Real and Complex Analysis, McGraw-Hill, 1970. 
[Shishikura 1987] M. Shishukura: On the quasiconformal surgery of rational functions, Ann. scient. Éc. Norm. Sup., $4^{e}$ série, t. 20 (1987),169.

[Shishikura] M. Shishukura: The connectivity of the Julia set and fixed points, Preprint IHES(1990), M/90/37. 


\section{LEBENSLAUF}

\section{Persönliche Daten}

Vorname: Ghazaleh

Nachname: $\quad$ Arghanoun

Geburtsdatum: $\quad$ 22. 10.1971

Geburtsort: $\quad$ Teheran, Iran.

Schule

10/1977 - 08/1985 Grund- und Mittelschule in Teheran, Iran.

10/1985 - 08/1989 Gymnasium in Teheran und Abitur im Fach Mathematik-Physik mit der Gesamtnote 18.43 („sehr gut“).

\section{Studium}

10/1989 - 07/1994 Aufnahmeprüfung und Studium der reinen Mathematik mit dem Ziel Bachelor of Science (B. Sc.) an der Sharif University of Technology in Teheran, Iran.

$08 / 1994$

Bachelor of Science in reiner Mathematik mit der Note 13.26 („befriedigend“) an der Sharif University of Technology, Teheran, Iran.

10/1995 - 02/1998 Aufnahmeprüfung und Studium der reinen Mathematik mit dem Ziel Master of Science (M. Sc.) an der Shahid Beheshti University in Teheran, Iran.

$07 / 1998$

Master of Science (M. Sc.) in reiner Mathematik mit der Note 17.71 (,sehr gut") an der Shahid Beheshti University, Teheran, Iran.

10/1999 - 09/2003 Förderung durch den Deutschen Akademischen Austauschdienst (DAAD) zur Promotion an der Georg-August-Universität in Göttingen, Deutschland, als Doktorandin von P.D. Dr. H. Kriete und Prof. Dr. M. Denker mit einer Dissertation zu komplexen dynamischen Systemen.

04/2004 Disputation am Mathematischen Institut der Georg-AugustUniversität, Göttingen. Der Titel der Doktorarbeit in reiner Mathematik lautet: Random Iterations of Subhyperbolic Relaxed Newton's Methods.

\section{Wissenschaftliche Aktivitäten}

$06 / 2001$

Vortrag mit dem Titel Random Iterations combined with the Newton's Method auf der Tagung "Tag der Funktionentheorie“ im Mathematischen Seminar der Christian-Albrecht-Universität zu Kiel. in Ecole normale superieure de Lyon, Lyon, Frankreich. 
Besuch der "Sissa-Prodyn Winter School: Geometric, Probabilistic and Variational Methods in Dynamical Systems" und im Anschluss daran Vortrag mit dem Titel Iterations of Special Rational Functions in Sissa: the International School for Advanced Studies in Trieste, Italien.

Seit 10/2004 Wissenschaftliche Mitarbeiterin an der Universität Mannheim. 